INTERNATIONAL

FOOD POLICY

RESEARCH

INSTITUTE

IFPRI

IFPRI Discussion Paper 01463

September 2015

\title{
Happiness in the Air
}

How Does a Dirty Sky Affect Subjective Well-being?

\section{Xin Zhang}

Xiaobo Zhang

Xi Chen

Development Strategy and Governance Division 


\section{INTERNATIONAL FOOD POLICY RESEARCH INSTITUTE}

The International Food Policy Research Institute (IFPRI), established in 1975, provides evidence-based policy solutions to sustainably end hunger and malnutrition and reduce poverty. The Institute conducts research, communicates results, optimizes partnerships, and builds capacity to ensure sustainable food production, promote healthy food systems, improve markets and trade, transform agriculture, build resilience, and strengthen institutions and governance. Gender is considered in all of the Institute's work. IFPRI collaborates with partners around the world, including development implementers, public institutions, the private sector, and farmers' organizations, to ensure that local, national, regional, and global food policies are based on evidence. IFPRI is a member of the CGIAR Consortium.

\section{AUTHORS}

Xin Zhang is a PhD candidate in the National School of Development at Peking Univeristy.

Xiaobo Zhang is a senior research fellow in the Development Strategy and Governance Division of the International Food Policy Research Institute, Washington, DC and a distinguished professor of economics at the National School of Development at Peking University.

Xi Chen (xi.chen@yale.edu) is an assistant professor at the School of Public Health and Department of Economics at Yale University and a research fellow at the Institute for the Study of Labor, Bonn, Germany.

\footnotetext{
Notices

1. IFPRI Discussion Papers contain preliminary material and research results and are circulated in order to stimulate discussion and critical comment. They have not been subject to a formal external review via IFPRI's Publications Review Committee. Any opinions stated herein are those of the author(s) and are not necessarily representative of or endorsed by the International Food Policy Research Institute.

2. The boundaries and names shown and the designations used on the map(s) herein do not imply official endorsement or acceptance by the International Food Policy Research Institute (IFPRI) or its partners and contributors.

Copyright 2015 International Food Policy Research Institute. All rights reserved. Sections of this material may be reproduced for personal and not-for-profit use without the express written permission of but with acknowledgment to IFPRI. To reproduce the material contained herein for profit or commercial use requires express written permission. To obtain permission, contact ifpri-copyright@cgiar.org.
} 


\section{Contents}

Abstract $\quad$ V

Acknowledgments $\quad$ vi

1. Introduction 1

2. Data 3

3. Empirical Strategy $\quad 6$

4. Results $\quad 9$

5. Implications for the Easterlin Paradox 23

6. Conclusion $\quad 25$

Appendix A: Supplementary Figures and Tables 26

Appendix B: Center for Epidemiologic Studies Depression scale (CES-D) in China Family Panel Studies (CFPS) 33

Appendix C: Sunshine Duration Calculation Formula 34

$\begin{array}{ll}\text { References } & 35\end{array}$ 


\section{Tables}

3.1 Summary statistics of key variables $\quad 7$

4.1 Effect of air quality (visibility versus API) on life satisfaction, longitudinal 10

4.2 Effect of air quality (visibility versus API) on hedonic happiness, cross-sectional 11

4.3 Effect of air quality (visibility versus API) on mental well-being, longitudinal 12

4.4 Effect of air quality (visibility versus API) on depressive symptoms, cross-sectional 14

4.5 Effect of air quality (visibility versus API) on severe depression, cross-sectional 15

4.6 Deviation-from-the-mean effect of air quality on life satisfaction 16

4.7 Heterogeneity test of air quality effect, by gender and age 17

4.8 Heterogeneity test of air quality effect, by income levels 18

4.9 Heterogeneity test of air quality effect, by pollution attitude and workplace 19

4.10 Heterogeneity test of air quality effect, by pollution level and health 20

4.11 Heterogeneity test of air quality effect, by education years 21

A.1 Heterogeneity test of air quality effect, by gender and age 30

A.2 Summary statistics of subjective well-being in China, 1990-2012 31

A.3 Placebo test: Air visibility forwarded by a week 32

\section{Figures}

2.1 Daily API and air visibility in China, 2010-2012 4

3.1 Relationship between visibility and subjective well-being 8

A.1 Happiness trend according to the China Central Television (CCTV) Postcard Survey, 2007-2012 26

A.2 Visibility trend in China, 1997-2012 26

A.3 Relation between visibility and $\mathrm{PM} 10, \mathrm{SO}_{2}$, and $\mathrm{NO}_{2}$

A.4 Interview date distribution, 2010 and 2012

A.5 The Baidu Index of “wumai (haze)" and "kouzhao (mask)", 2012-2013 28

A.6 Daily API in China, 2010-2012 29

C.1 Sunshine duration calculation $\quad 34$ 


\begin{abstract}
Existing studies that evaluate the impact of pollution on human beings understate its negative effect on cognition, mental health, and happiness. This paper attempts to fill in the gap via investigating the impact of air quality on subjective well-being using China as an example. By matching a unique longitudinal dataset at the individual level, which includes self-reported happiness and mental well-being measures, with contemporaneous local air quality and weather information according to the exact date and place of interview, we show that worse air quality reduces shorter-term hedonic happiness and increases the rate of depressive symptoms. However, life satisfaction, an evaluative measure of happiness, is largely immune from immediate bad air quality.
\end{abstract}

Keywords: hedonic happiness, life satisfaction, mental well-being, air quality, China

JEL Codes: 131, Q51, Q53 


\section{ACKNOWLEDGMENTS}

We are grateful for being given access to the data of China Family Panel Studies (CFPS), which is funded by 985 Program of Peking University and carried out by the Institute of Social Science Survey of Peking University. Xi Chen acknowledges financial support from the James Tobin Summer Research Fund at Yale Economics Department, a NIH/NIA grant (\# 1 R03 AG048920-01), and the Yale Macmillan Center Faculty Research Award on air pollution and health. This paper was undertaken as a part of the CGIAR Research Program on Policies, Institutions, and Markets (PIM) and Agriculture for Nutrition and Health (A4NH), both led by the International Food Policy Research Institute (IFPRI). This paper has not gone through IFPRI's standard peer-review procedure. The opinions expressed here belong to the authors, and do not necessarily reflect those of PIM, A4NH, IFPRI, or CGIAR. 


\section{INTRODUCTION}

It has been well documented that exposure to air pollution increases health risks, such as cardiovascular diseases (Gallagher et al. 2010), respiratory diseases (Beatty and Shimshack 2014), and even mortality (Chen et al. 2013). However, less is known about how air pollution affects the subjective dimension of well-being. Studies on the latter aspect have the potential to improve happiness, arguably the ultimate goal of human beings.

Among the few existing studies on this linkage between air pollution and happiness, most rely on either aggregate air pollution and happiness data (Menz 2011) or cross-sectional individual-level data (Levinson 2012). Studies based on aggregated data, however, are subject to the ecological fallacy. That is, the findings may differ or even contradict each other depending on the levels of aggregation. Studies at the individual level on the basis of cross-sectional data, on the other hand, are prone to estimation biases because not all the individual-specific factors can be taken into account.

Moreover, almost all the studies use air quality data averaged over a rather long period, such as one year (Ferreira et al. 2013). The average air quality data from a long span may differ from the actual data on the day of the interview, which should more directly affect interviewees' responses to subjective well-being (SWB) questions. The associated measurement errors of air quality also could result in biased estimates.

Finally, the literature primarily uses life satisfaction, an evaluative measure of SWB, or makes no explicit distinction between hedonic and evaluative measures (Levinson 2012). Hedonic happiness refers to moment-to-moment experienced utility and directly links to immediate emotions and affection, while evaluative happiness reflects an overall assessment of life. As noted in Kahneman and Deaton (2010) and Deaton and Stone (2013), the two measures could yield drastically different results because they are often influenced by different factors.

To overcome these problems, we employ a novel national longitudinal survey of individuals, with rich measures of both hedonic and evaluative happiness, in combination with contemporaneous local air quality and weather information at the time and location of the interview. The matched longitudinal data enable us to remove individual unobserved factors, thus largely alleviating omitted variable biases. The local air quality on the day of the interview can more precisely reflect environmental amenities facing interviewees than the heretofore more often used measure of average air quality during a long span.

Based on the matched longitudinal dataset of SWB and daily air quality, we find that lower air visibility significantly reduces shorter-term hedonic happiness and raises the rate of depressive symptoms. The impact on life satisfaction, however, is largely muted, possibly due to hedonic adaptation over time. An increase of 1 standard deviation $(S D)$ in air visibility boosts hedonic happiness (scaled from 1 to 4 ) by 0.043 , slightly less than the impact of relative income status (0.064), which is one of the most important determinants of happiness in the literature. A back-of-the-envelope analysis indicates that the decline in visibility accounts for 6.6 percent of the actual decline in happiness during the period from 1997 through 2012. Putting this into monetary terms, people on average are willing to pay CNY 2.9 per day per person (more than US\$170 per year per person) for a $0.1 S D$ improvement in air quality. ${ }^{1}$

To the best of our knowledge, this is the first study examining the impact of air quality on SWB in a developing country. Compared to air pollution in developed countries, air pollution is generally more serious, sometimes even life threatening, in developing countries (Tanaka 2012; Chen et al. 2013; Greenstone and Hanna 2014). There is thus a need for more empirical evidence on the impact of air quality on human welfare.

Our study also is related to a few strands of literature. It contributes to the debate about the Easterlin paradox, that is, the observation that over time happiness does not go up despite income growth. Few studies have explained the puzzle from the viewpoint of air quality, which is a focus of our paper. It also adds to the burgeoning body of literature on the impact of air pollution on various outcomes, such as

\footnotetext{
${ }^{1}$ All dollars are US dollars.
} 
standardized test scores, human capital formation, later labor market outcomes (Lavy, Ebenstein, and Roth 2014a, 2014b), and the productivity of indoor workers (Chang et al. 2014; Li, Liu, and Salvo 2015). Long-term exposure to air pollution may even change the structure and function of brains (Weir 2012). The associated long-term economic consequences identified in the literature and the sizable willingnessto-pay (WTP) to improve air quality from our back-of-the-envelope calculation warn us that careful evaluations of the impact of air pollution on SWB are necessary.

The reminder of the paper is organized as follows. Section 2 describes the data. Section 3 lays out the empirical strategy. Section 4 presents our main findings, including heterogeneity tests. Section 5 discusses implications for the Easterlin paradox. Finally, Section 6 concludes. 


\section{DATA}

\section{Subjective Well-being (SWB)}

For happiness measures, we rely on the China Family Panel Studies (CFPS), a nationally representative survey of Chinese communities, families, and individuals conducted in 2010 and 2012. The CFPS is funded by Peking University and carried out by the Institute of Social Science Survey of Peking University. The CFPS covers a wide range of domains for families and individuals from 162 counties in 25 provinces of China, including their economic activities, education outcomes, family dynamics and relationships, health, and SWB.

There are four advantages of the CFPS for our purposes. First, information about geographic locations and dates of interviews for all respondents enables us to precisely match individual happiness measures in the survey with external air quality data. Second, rich measures of happiness ranging from moment-to-moment happiness to mental well-being and life satisfaction allow us to compare the effects of air pollution in various time frames. Third, the longitudinal data allow us to remove unobserved individual factors that may bias the results. Fourth, the survey collected rich information at multiple levels, allowing us to control for a wide range of covariates.

We make use of three types of SWB measures. The first is life satisfaction (Welsch 2006, 2007; Rehdanz and Maddison 2008; MacKerron and Mourato 2009; Luechinger 2009, 2010; Menz 2011; Ferreira et al. 2013). Both the CFPS 2010 and the CFPS 2012 asked each respondent to answer the question, "Overall, how satisfied are you with your life?" on a scale from 1 (not satisfied at all) to 5 (very satisfied). Panels are constructed to implement individual fixed effect estimations. With no specified time frame, life satisfaction, an evaluative measure of SWB, reflects the extent to which people's own experiences match their long-term aspirations and expectations about their lives as a whole (Stone and Mackie 2014). Life circumstances, such as income, education, and social status, are among the main determinants of life satisfaction.

The second SWB measure is the Center for Epidemiologic Studies Depression scale (CES-D), a self-reported scale designed to measure the current level of depressive symptomatology in the general population. Compared to life satisfaction, the CES-D highlights emotional experiences that affect people during a shorter period.

The CFPS 2012 uses a standard 20-item scale developed by Radloff (1977) (Appendix B). There are four options for each item, scaled from 0 (rarely or none of the time) to 3 (most or all of the time). Four items (4th, 8th, 12th, and 16th) worded in inverse order in the CES-D are transformed back to be consistent with other items. The total CES-D score is valued between 0 and 60 , with higher scores indicating more negative symptoms during the past week. Along with the continuous CES-D score, two cut-off scores indicate depression, namely, depressive symptoms (for example, 16 or greater) and severe depressive symptoms (for example, 21 or greater) (Radloff 1977; Bailly, Beuscart, and Collinet 1992).

However, the CFPS 2010 implements only a short form six-item CES-D test (Appendix B). We therefore construct a balanced panel and generate the percentile of the total CES-D score, respectively, for CFPS 2010 and CFPS 2012 for each respondent, which helps link the two waves of survey data in the longitudinal analysis.

The third SWB measure gauges short-term hedonic happiness. The CFPS 2012 asked respondents to answer the question, "I was happy" with a number between 1 and 4 . The higher the number, the happier the respondent was during the past week. Compared to life satisfaction, CES-D score and hedonic happiness are more directly related to the environment and people's affective state in day-to-day and moment-to-moment life (Stone and Mackie 2014). ${ }^{2}$

\footnotetext{
${ }^{2}$ Hedonic well-being is closely related to the often-used terms "experienced well-being" and "emotional well-being." They are often used interchangeably in the literature.
} 


\section{Air Quality}

We use two measures of air quality, the air pollution index (API) and air visibility, which move in opposite directions (Figure 2.1). The first measure, API, ranges from 0 to 500, and a larger value indicates worse air quality. API is generated by a piecewise linear transformation from the concentrations of three air pollutants, including sulfur dioxide $\left(\mathrm{SO}_{2}\right)$, nitrogen dioxide $\left(\mathrm{NO}_{2}\right)$, and fine particulate matter smaller than 10 micrometers (PM10). Evidence suggests that fine particulate matter is detrimental to health and human capital (Cohen et al. 2005; Lavy, Ebenstein, and Roth 2014a, 2014b) and is of high concentration in China (Li, Liu, and Salvo 2015).

Daily observations of API are obtained from the air quality report published by the Ministry of Environmental Protection of China. The report, which started in June 2000 and covered 120 major cities in 2012, involves all the provincial municipalities and provincial capitals. However, our knowledge of API and composition of air pollution relies mainly on daily reports, which were not on the radar of public media during the CFPS survey period (2010-2012). For example, keyword searches of "wumai (haze)" and "kouzhao (mask)" using China's major search engine, baidu.com, indicate that public awareness did not spike until a major crisis of thick haze covering much of eastern China in early 2013 (Figure A.5). Moreover, it is concerning that API reports by the local government are less reliable than external sources, especially due to the possible manipulation of daily API to reach the standard of a "blue-sky day" (Chen et al. 2012; Ghanem and Zhang 2014).

The second measure, air visibility, is defined as the greatest distance at which an observer can just see a black object viewed against the horizon (Malm 1999). Atmospheric researchers confirm visibility is a good predictor of main air pollutant concentrations, such as fine particulate matter, $\mathrm{SO}_{2}$, and $\mathrm{NO}_{2}($ Lee and Sequeira 2001; Qiu and Yang 2000; Cheung et al. 2005; Deng et al. 2008). ${ }^{3}$

\section{Figure 2.1 Daily API and air visibility in China, 2010-2012}

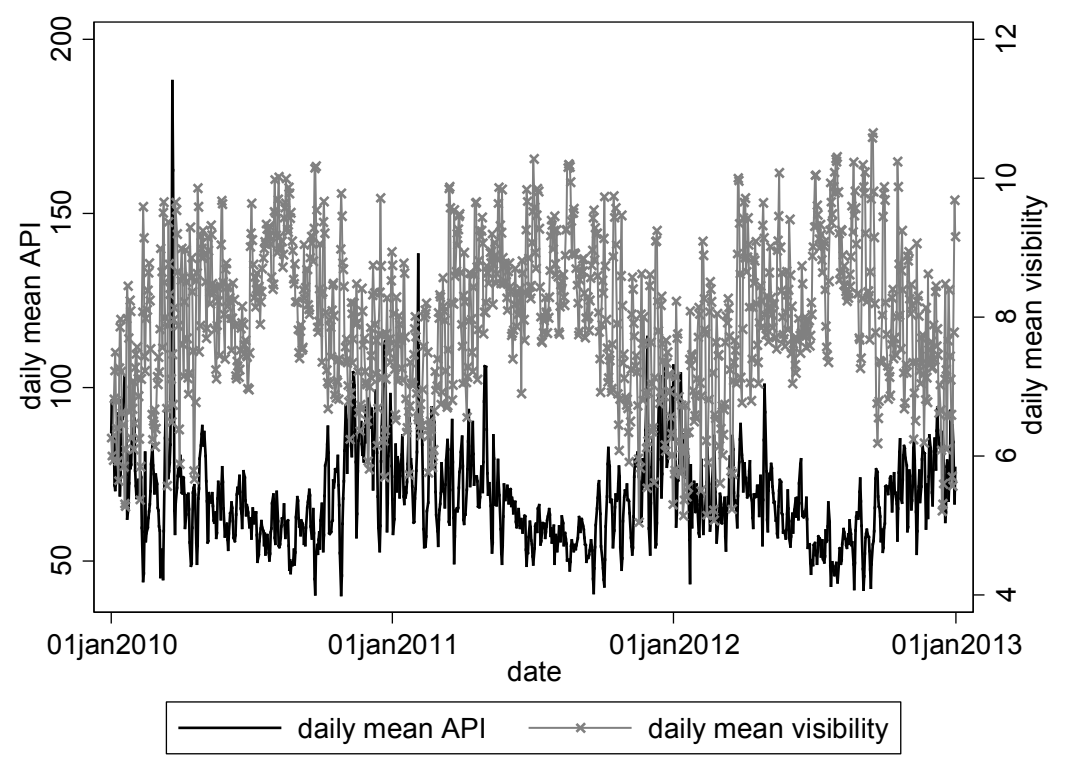

Source: National Oceanic and Atmospheric Administration (2010-2012); Ministry of Environmental Protection of China (2010 2012).

Note: API = air pollution index. The daily mean API is calculated by the weighted average values of all the API report cities in China, where the weights are the yearly population in each city. The daily mean visibility is calculated by the weighted average values of all the monitor stations in China, where the weights are equal to the yearly population at the county level.

\footnotetext{
${ }^{3}$ See Figure A3 for an example of Lanzhou, the capital city of Gansu province, from 2009 to 2012, where individual pollutant components are available. Visibility is observed to be highly negatively correlated with the three main pollutants, indicating air visibility is a good proxy for air quality.
} 
Monitored by the National Climatic Data Center of the National Oceanic and Atmospheric Administration, air visibility complements API in measuring air quality. First, air visibility is much easier to perceive than the colorless components in the API, including $\mathrm{SO}_{2}$ and $\mathrm{NO}_{2}$. Second, this external source of air quality data is less likely to be manipulated and is therefore more reliable. Third, relative to API, visibility stations are more evenly distributed in China during a longer period, and therefore air visibility is more nationally representative and better matches the CFPS surveys. Daily air visibility records are from 400 monitor stations in China between 2009 and 2012, much larger than the number of monitor stations for API. Fourth, the longitudes and latitudes of visibility monitor stations facilitate us to more precisely measure distances away from monitors. Fifth, the air visibility data record rich weather conditions, such as temperature, precipitation, wind speed, and indicators for bad weather, ${ }^{4}$ which isolates the impact of air quality from weather patterns and therefore mitigates potential estimation biases.

To merge the CFPS survey with air visibility and weather data, we calculate a weighted average value of all the monitor stations within 60 kilometers of the centroid of each CFPS surveyed county, where the weights are equal to the inverse of the square root of the distance between the monitor stations and county centroids. ${ }^{5,6}$ Meanwhile, we match each CFPS county to the nearest city with an API report. ${ }^{7}$

The CFPS surveyed a balanced panel of 22,429 individual respondents (or 44,858 observations) in 2010 and 2012, of which 29,830 observations could be matched to air visibility and weather data within 60 kilometers of the county centroids. Among the 29,830 observations, API readings are missing for 3,065 observations when matched with the air quality report published by the Ministry of Environmental Protection of China in 2010, and self-rated relative income statuses are missing for 2,381 observations. The final dataset for analyses includes 27,433 observations and 24,753 observations after merging with the visibility data and the API data, respectively. ${ }^{8}$ The differences in sample size among regressions are due to a small but different number of missing values for various SWB measures.

\footnotetext{
${ }^{4}$ Bad weather includes fog, rain or drizzle, snow or ice pellets, hail, thunder, and tornadoes or funnel clouds.

${ }^{5}$ One exception is that the bad weather indicator is matched to the nearest monitor to each county.

${ }^{6}$ Our baseline results are robust to matching using narrower radiuses (for example, 50 kilometers) and alternative weights (for example, inverse of the distance or squared distance between the monitor stations and the county centroids).

${ }^{7}$ We do not calculate weighted air pollution index (API) for two reasons: one, API depends on the local dominant pollutant, and two, the officially released API data are from far fewer monitor stations than air visibility data.

${ }^{8}$ A balance test shows that there are no significant differences of key characteristics between the matched and unmatched samples, mitigating the concern for selection bias in our matching process.
} 


\section{EMPIRICAL STRATEGY}

Our baseline econometric specification is as follows:

$$
\begin{aligned}
H_{i j t}= & \alpha_{1} \ln P_{j t}+\alpha_{2} \ln \text { sunshine }_{j t}+\alpha_{3} \ln P_{j t} \times \ln \text { sunshine }_{j t} \\
& +\beta_{1} \ln Y_{i j t}+\beta_{2} R_{i j t}+X_{i j t}^{\prime} r+W_{j t}^{\prime} \phi+\lambda_{i}+\delta_{j}+\eta_{t}+\varepsilon_{i j t}
\end{aligned}
$$

The dependent variable $H_{i j t}$ is the self-reported happiness of respondent $i$ in county $j$ at date $t . \ln P_{j t}$ is the $\log$ form of air quality measure in county $j$ at date $t$. We add lnsunshine $e_{j}$, the log form of sunshine duration (hours) in county $j$ at date $t$, as a key explanatory variable. Studies show that sunshine affects individuals' moods, social behavior, and health. For example, sunshine can significantly increase people's willingness to help and generosity of tips (Cunningham 1979). People born in the winter suffer a higher risk of schizophrenia than those born in sunnier months (Wolfson 2013). Though there is no record of sunshine hours, we calculate approximate hours of sunshine in county $j$ at date $t$ according to the latitude of county $j$ and the latitude of direct sunshine point at date $t$ (Appendix C). $\ln P_{j t} \times \ln s u n s h i n e_{j t}$, an interaction term between $\ln P_{j t}$ and $\ln$ sunshine $_{j t}$ captures the interactive effect between air quality and hours of sunshine.

We test whether air quality has a positive effect on SWB. However, it is likely that people may prefer a less clear sky on hot summer days with longer hours of sunshine. Because most of the CFPS interviews were conducted during the summer when college students took advantage of their summer vacation to finish the fieldwork (Figure A.4), it is necessary to test whether longer hours of sunshine weaken the positive effect of clear skies on SWB. Specifically, when $P_{j t}$ represents air visibility and $H_{i j t}$ denotes life satisfaction or hedonic happiness, we hypothesize that $\alpha_{1}$ is significantly positive and $\alpha_{3}$ is significantly negative.

We control for the $\log$ form of absolute household per capita income $\ln Y_{i j t}$, the self-rated relative income status $R_{i j t}$ ranging from 1 (lowest) to 5 (highest); a set of demographic correlates of happiness $X_{i j t}$, including age and its square term; gender; marital status; ${ }^{9}$ years of education; occupation; unemployment status; party membership; and health status (Oswald 1997; Knight, Song, and Gunatilaka 2009; Knight and Gunatilaka 2010, 2011; Easterlin et al. 2012) and a vector of weather conditions $W_{j t}$, involving mean temperature and its square term; daily maximum-minimum temperature gap; total precipitation; maximum sustained wind speed; and a dummy for bad weather on the day of observation. We control for rich weather conditions to rule out the possibility that they are correlated with both SWB and air quality and therefore bias our results. The daily maximum-minimum temperature gap serves as a proxy for cloud cover of the day since a smaller gap usually indicates a higher chance of cloudy weather (Mearns and Best 2013). Controlling for the temperature gap also may mitigate potential biases of theoretical sunshine hours and visibility due to cloud cover. $\lambda_{i}$ denotes individual fixed effect; $\delta_{j}$ represents county fixed effect; $\eta_{t}$ indicates month, year, and day-of-week fixed effects; and $\varepsilon_{i j t}$ is the error term. Standard errors are clustered at the county level. Table 3.1 describes key variables and their summary statistics.

\footnotetext{
${ }^{9}$ Marital status includes those never married, married, and divorced. We set those never married as the reference group.
} 
Table 3.1 Summary statistics of key variables

\begin{tabular}{|c|c|c|c|c|c|}
\hline \multirow[b]{2}{*}{ Variable } & \multirow[b]{2}{*}{ Definition } & \multicolumn{2}{|c|}{2010} & \multicolumn{2}{|c|}{2012} \\
\hline & & Mean & $\begin{array}{l}\text { Standard } \\
\text { deviation }\end{array}$ & Mean & $\begin{array}{l}\text { Standard } \\
\text { deviation }\end{array}$ \\
\hline $\begin{array}{l}\text { Life } \\
\text { satisfaction }\end{array}$ & $\begin{array}{l}\text { Life satisfaction, ranging from } 1 \text { to } 5 \text {, the } \\
\text { higher the better }\end{array}$ & 3.489 & 1.040 & 3.321 & 1.058 \\
\hline $\begin{array}{l}\text { Hedonic } \\
\text { happiness }\end{array}$ & $\begin{array}{l}\text { Answer to the question "I was happy," } \\
\text { ranging from } 1 \text { to } 4 \text {, the higher the better }\end{array}$ & - & - & 2.811 & 1.000 \\
\hline CES-D score & $\begin{array}{l}\text { The total score of the CES-D, } 0-60 \text {, the } \\
\text { lower the better }\end{array}$ & 3.074 & 3.876 & 13.059 & 8.077 \\
\hline $\begin{array}{l}\text { Depressive } \\
\text { symptoms }\end{array}$ & $\begin{array}{l}\text { Indicator for depressive symptoms (= } 1 \text { if } \\
\text { CES-D score } \geq 16 \text { ) }\end{array}$ & - & - & 0.326 & 0.469 \\
\hline $\begin{array}{l}\text { Severe } \\
\text { depression }\end{array}$ & $\begin{array}{l}\text { Indicator for severe depression (= } 1 \text { if CES- } \\
D \text { score } \geq 21 \text { ) }\end{array}$ & - & - & 0.166 & 0.373 \\
\hline $\begin{array}{l}\text { Mental well- } \\
\text { being }\end{array}$ & The percentile of the CES-D score & 0.500 & 0.289 & 0.500 & 0.289 \\
\hline Visibility & log form of Weighted visibility (miles) & 8.505 & 4.153 & 8.783 & 4.064 \\
\hline $\mathrm{API}$ & log form of air pollution index & 64.463 & 35.998 & 58.857 & 21.083 \\
\hline Sunshine & Sunshine hours & 13.254 & 0.780 & 12.195 & 1.356 \\
\hline $\begin{array}{l}\text { Per capita } \\
\text { income }\end{array}$ & $\begin{array}{l}\text { Log form of household per capita income (in } \\
\text { Chinese yuan) }\end{array}$ & 8.620 & 1.056 & 8.990 & 1.128 \\
\hline $\begin{array}{l}\text { Relative } \\
\text { income }\end{array}$ & $\begin{array}{l}\text { Self-rated relative income status, the higher } \\
\text { the better }\end{array}$ & 2.203 & 0.970 & 2.232 & 0.977 \\
\hline Temperature & Weighted mean temperature $\left(10^{\circ} \mathrm{F}\right)$ & 7.389 & 1.109 & 6.693 & 2.211 \\
\hline $\begin{array}{l}\text { Temp. } \\
\text { squared }\end{array}$ & $\begin{array}{l}\text { Weighted mean temperature }\left(10^{\circ} \mathrm{F}\right) \\
\text { squared }\end{array}$ & 55.830 & 14.770 & 49.681 & 23.073 \\
\hline Temp. diff. & $\begin{array}{l}\text { Temperature difference (daily weighted } \\
\text { maximum-minimum, } 10^{\circ} \mathrm{F} \text { ) }\end{array}$ & 1.532 & 0.618 & 1.450 & 0.540 \\
\hline Precipitation & Weighted total precipitation (inches) & 0.164 & 0.465 & 0.139 & 0.395 \\
\hline Wind speed & $\begin{array}{l}\text { Weighted maximum sustained wind speed } \\
\text { (knots) }\end{array}$ & 8.460 & 3.845 & 8.945 & 4.059 \\
\hline Bad weather & $\begin{array}{l}\text { Indicator for bad weather (fog, rain or } \\
\text { drizzle, snow or ice pellets, hail, thunder, } \\
\text { tornadoes or funnel clouds) }\end{array}$ & 0.471 & 0.499 & 0.439 & 0.496 \\
\hline Age & Age $(\div 10)$ & 4.657 & 1.530 & 4.856 & 1.530 \\
\hline Age squared & Age $(\div 10)$ squared & 24.027 & 14.609 & 25.918 & 15.206 \\
\hline Male & Indicator for males & 0.480 & 0.500 & 0.480 & 0.500 \\
\hline Married & Indicator for married status & 0.842 & 0.365 & 0.841 & 0.365 \\
\hline Divorced & Indicator for divorced status & 0.012 & 0.107 & 0.014 & 0.117 \\
\hline Education & years of education & 6.098 & 4.898 & 6.705 & 4.856 \\
\hline Unemployed & Indicator for unemployed status & 0.060 & 0.238 & 0.011 & 0.104 \\
\hline $\begin{array}{l}\text { State } \\
\text { employee }\end{array}$ & $\begin{array}{l}\text { Indicator for working in the government, } \\
\text { public institutions, or SOEs }\end{array}$ & 0.067 & 0.250 & 0.080 & 0.272 \\
\hline Party & Indicator for party membership & 0.077 & 0.267 & 0.084 & 0.278 \\
\hline $\begin{array}{l}\text { Chronic } \\
\text { disease }\end{array}$ & Indicator for suffering from chronic diseases & 0.152 & 0.359 & 0.141 & 0.348 \\
\hline
\end{tabular}

Source: China Family Panel Studies (ISSS 2010; 2012).

Note: $\quad$ API = air pollution index; CES-D = Center for Epidemiologic Studies Depression scale; SOEs = state-owned enterprises. Dashes indicate no data for the 2010 wave of survey. The summary statistics are calculated based on the matched and balanced panel data. 
Figure A.4 shows the distribution of interview dates for the two waves of the CFPS national sample, which span all months and seasons and thus enable us to isolate the impact of air pollution from seasonality. Overall, a majority of surveys were conducted in summer or winter as those seasons largely overlap with the summer vacation and winter break of the college students who implemented the CFPS. Variations for identification in our individual fixed effect model (equation 1) come from differential exposure to air pollution for the same respondent across the two waves. The average absolute change in visibility for each individual across waves amounts to 2.83 miles. For outcome indicators available in only one of the two waves, such as hedonic happiness, our estimation strategy relies on differential exposure to air pollution for different respondents living in the same county in the same wave of the survey.

Before undertaking quantitative analyses, we plot the relationships between air visibility and main SWB measures (Figure 3.1). We first generate the visibility residuals by regressing visibility on county fixed effects, year and month fixed effects, and rich weather conditions. We plot the mean of visibility residuals within each level of life satisfaction, CES-D score, and hedonic happiness. As shown in Figure 3.1, life satisfaction and hedonic happiness demonstrate a positive relationship with air visibility, while CES-D score has a negative relationship, in accordance with our expectation. While these bivariate plots provide some suggestive evidence, we present a more rigorous analysis in the next section.

Figure 3.1 Relationship between visibility and subjective well-being
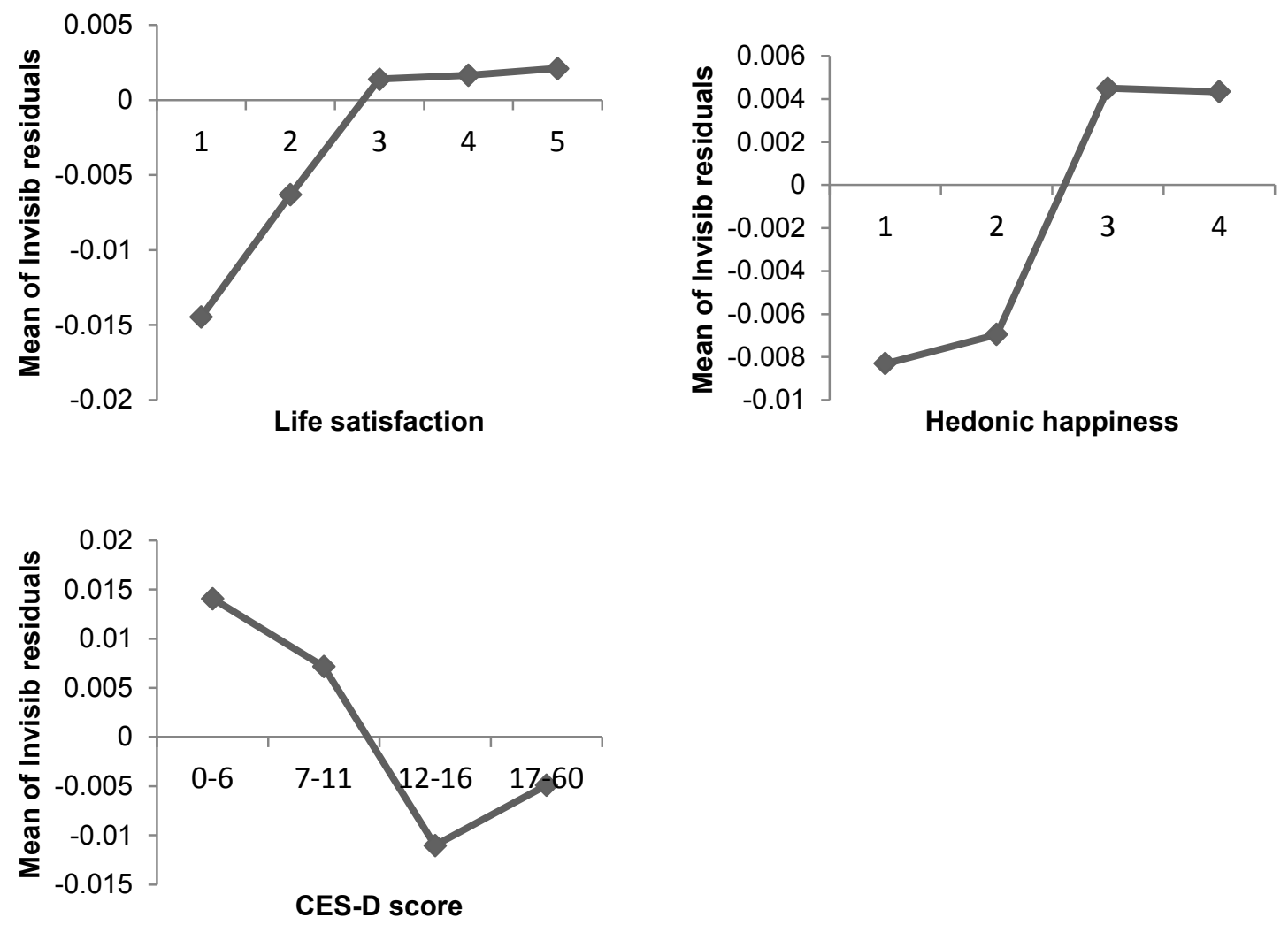

Source: Life satisfaction is plotted from the China Family Panel Studies (ISSS 2010; 2012). Hedonic happiness and CES-D score are plotted from China Family Panel Studies (ISSS 2012).

Note: $\quad$ CES-D = Center for Epidemiologic Studies Depression scale. The visibility residuals are generated by regressing visibility on county fixed effects, year and month fixed effects, and weather controls. 


\section{RESULTS}

\section{Baseline Results}

We report baseline results on air quality and various SWB measures. Columns (4) and (8) of Tables 4.1 through 4.5 present results from our preferred specifications.

Table 4.1 presents longitudinal evidence on life satisfaction. Columns (1) through (4) approximate air quality by air visibility. Column (1) identifies the effect of air quality while controlling for demographic characteristics and individual fixed effects. We do not find a significant relationship between daily air quality and life satisfaction. Relative income imposes a highly significant effect on life satisfaction, while there is no significant effect for absolute income. People who are in poor health or divorced tend to have lower life satisfaction. Column (2) further adds sunshine hours and its interaction with visibility. The coefficient of air visibility remains insignificant, as do the coefficients on sunshine hours and the interaction term. Moreover, our main results stay robust when rich weather conditions and year, month, and day-of-week fixed effects are further controlled for in columns (3) and (4).

Columns (5) through (8) repeat the exercises in columns (1) through (4), using API as a measure of air quality. The coefficients of API and its interaction with sunshine remain insignificant in all the specifications, suggesting that short-term air quality has little impact on long-term life evaluation.

Table 4.2 examines the effect of air quality on hedonic happiness. Since hedonic happiness was surveyed only in the CFPS 2012, we report cross-sectional evidence with county fixed effects in all specifications. Both higher air visibility and lower API significantly improve hedonic happiness. Consistent with the happiness literature, there is a U-shaped relationship between age and hedonic happiness, and the trough of hedonic happiness is around age 61. Married men who receive more education and are healthier tend to be happier. Party membership is associated with being happier, presumably because it symbolizes higher social status and social recognition.

Column (2) of Table 4.2 adds sunshine hours of the day and its interaction with air visibility. As expected, hedonic happiness increases with both visibility and hours of sunshine on the day of interview but decreases with the interaction between visibility and sunshine. Columns (3) and (4) further control for seasonality and weather-related effects by including rich weather conditions and month and day-of-week fixed effects. Our main results remain robust to these additional controls. The insignificant sunshine effect in column (4) may be due to its colinearity with month fixed effects.

Columns (5) through (8) of Table 4.2 re-estimate columns (1) through (4) after replacing air visibility with API and generate consistent results. Specifically, API has a significant and negative effect on hedonic happiness, and the effect is partially offset by long hours of sunshine. Therefore, evidence from both China-monitored API data and US-monitored air visibility data suggests a salient impact of air quality on hedonic happiness.

Though only a six-item CES-D was collected in the CFPS 2010, rendering it incompatible with the 20-item CES-D in the CFPS 2012, we restrict the analysis to the balanced panel and transform CES-D scores in each wave to their corresponding percentiles. The lower the percentile in the CES-D score distribution, the lower the chance of depression. Now we are able to implement all estimations in Table 4.3 with individual fixed effects as we did in Table 4.1. In column (1) of Table 4.3, the coefficient on visibility is negative but statistically insignificant, which becomes significant when hours of sunshine and the interaction term are included in columns (2) and (4). The effect of air visibility is robust to the inclusion of rich weather conditions and year, month, and day-of-week fixed effects. The results using API in columns (5) through (8) reveal the same pattern, further indicating that good air quality improves mental well-being. 
Table 4.1 Effect of air quality (visibility versus API) on life satisfaction, longitudinal

\begin{tabular}{|c|c|c|c|c|c|c|c|c|}
\hline \multirow{2}{*}{$\begin{array}{l}\text { Dependent variable } \\
\text { Life satisfaction }\end{array}$} & \multicolumn{4}{|c|}{ Visibility } & \multicolumn{4}{|c|}{ API } \\
\hline & (1) & (2) & (3) & (4) & (5) & (6) & $(7)$ & $(8)$ \\
\hline \multirow[t]{2}{*}{ Air quality } & 0.037 & 0.674 & 0.693 & 0.763 & -0.022 & -1.228 & -1.329 & -1.128 \\
\hline & $(0.026)$ & $(0.462)$ & $(0.579)$ & $(0.637)$ & $(0.042)$ & $(1.490)$ & $(1.287)$ & $(1.395)$ \\
\hline \multirow[t]{2}{*}{ Sunshine } & & 0.566 & $0.773^{*}$ & 0.706 & & -1.926 & -1.796 & -1.207 \\
\hline & & $(0.408)$ & $(0.463)$ & $(0.905)$ & & $(2.419)$ & $(2.137)$ & $(2.536)$ \\
\hline \multirow[t]{2}{*}{ Air quality $\times$ Sunshine } & & -0.253 & -0.260 & -0.286 & & 0.478 & 0.516 & 0.427 \\
\hline & & $(0.183)$ & $(0.228)$ & $(0.250)$ & & $(0.582)$ & $(0.503)$ & $(0.544)$ \\
\hline \multirow[t]{2}{*}{ Household per capita income } & 0.015 & 0.015 & 0.016 & $0.022^{*}$ & 0.017 & 0.016 & 0.017 & $0.025^{\star}$ \\
\hline & $(0.013)$ & $(0.013)$ & $(0.013)$ & $(0.012)$ & $(0.014)$ & $(0.014)$ & $(0.014)$ & $(0.013)$ \\
\hline \multirow[t]{2}{*}{ Relative income } & $0.203^{* * *}$ & $0.203^{* * *}$ & $0.204^{* * *}$ & $0.204^{* * *}$ & $0.204^{* * *}$ & $0.204^{* * *}$ & $0.205^{\star \star \star}$ & $0.204^{* * *}$ \\
\hline & $(0.012)$ & $(0.012)$ & $(0.012)$ & $(0.012)$ & $(0.014)$ & $(0.013)$ & $(0.013)$ & $(0.013)$ \\
\hline \multirow[t]{2}{*}{ Age $(\div 10)$ squared } & $-0.070^{* * *}$ & $-0.067^{* * *}$ & $-0.062^{* \star *}$ & 0.026 & $-0.077^{* * *}$ & $-0.073^{* * *}$ & $-0.070^{* * *}$ & 0.027 \\
\hline & $(0.011)$ & $(0.011)$ & $(0.012)$ & $(0.020)$ & $(0.012)$ & $(0.013)$ & $(0.014)$ & $(0.022)$ \\
\hline \multirow[t]{2}{*}{ Married } & 0.044 & 0.045 & 0.051 & $0.114^{*}$ & 0.021 & 0.017 & 0.024 & 0.094 \\
\hline & $(0.065)$ & $(0.065)$ & $(0.065)$ & $(0.066)$ & $(0.076)$ & $(0.074)$ & $(0.074)$ & $(0.074)$ \\
\hline \multirow[t]{2}{*}{ Divorced } & $-0.317^{* *}$ & $-0.312^{* *}$ & $-0.303^{\star *}$ & $-0.245^{*}$ & $-0.384^{* *}$ & $-0.380^{* *}$ & $-0.369^{* *}$ & $-0.296^{*}$ \\
\hline & $(0.142)$ & $(0.142)$ & $(0.142)$ & $(0.145)$ & $(0.154)$ & $(0.153)$ & $(0.153)$ & $(0.158)$ \\
\hline \multirow[t]{2}{*}{ Education } & -0.000 & -0.000 & -0.000 & 0.004 & -0.001 & -0.001 & -0.000 & 0.004 \\
\hline & $(0.006)$ & $(0.006)$ & $(0.006)$ & $(0.006)$ & $(0.007)$ & $(0.007)$ & $(0.007)$ & $(0.007)$ \\
\hline \multirow[t]{2}{*}{ Unemployed } & -0.003 & -0.004 & -0.006 & -0.041 & -0.022 & -0.029 & -0.031 & -0.070 \\
\hline & $(0.061)$ & $(0.061)$ & $(0.061)$ & $(0.059)$ & $(0.071)$ & $(0.069)$ & $(0.070)$ & $(0.066)$ \\
\hline \multirow[t]{2}{*}{ State employee } & 0.026 & 0.026 & 0.029 & 0.033 & 0.039 & 0.039 & 0.042 & 0.044 \\
\hline & $(0.048)$ & $(0.048)$ & $(0.047)$ & $(0.048)$ & $(0.055)$ & $(0.054)$ & $(0.054)$ & $(0.053)$ \\
\hline \multirow[t]{2}{*}{ Party } & 0.050 & 0.049 & 0.048 & 0.064 & 0.091 & 0.092 & 0.089 & 0.106 \\
\hline & $(0.068)$ & $(0.068)$ & $(0.068)$ & $(0.067)$ & $(0.071)$ & $(0.072)$ & $(0.071)$ & $(0.071)$ \\
\hline \multirow{2}{*}{ Chronic disease } & $-0.091^{* \star *}$ & $-0.091^{* * *}$ & $-0.092^{* * *}$ & $-0.092^{* * *}$ & $-0.095^{* * *}$ & $-0.096^{* * *}$ & $-0.097^{* * *}$ & $-0.097^{\star * *}$ \\
\hline & $(0.026)$ & $(0.026)$ & $(0.026)$ & $(0.026)$ & $(0.030)$ & $(0.031)$ & $(0.030)$ & $(0.030)$ \\
\hline Individual fixed effect & Yes & Yes & Yes & Yes & Yes & Yes & Yes & Yes \\
\hline Weather controls & No & No & Yes & Yes & No & No & Yes & Yes \\
\hline Year, month, day-of-week fixed effects & No & No & No & Yes & No & No & No & Yes \\
\hline Observations & 27,409 & 27,409 & 27,409 & 27,409 & 24,729 & 24,729 & 24,729 & 24,729 \\
\hline (Within) $R$-squared & .048 & .048 & .049 & .054 & .050 & .050 & .051 & .058 \\
\hline
\end{tabular}

Source: China Family Panel Studies (ISSS 2010; 2012).

Note: API = air pollution index. The weather controls include mean temperature and its square, temperature difference (daily maximum-minimum), total precipitation, maximum sustained wind speed, and a dummy for bad weather. Robust standard errors, clustered at the county level, are presented in parentheses. $* 10 \%$ significance level. **5\% significance level. $* * * 1 \%$ significance level. 
Table 4.2 Effect of air quality (visibility versus API) on hedonic happiness, cross-sectional

\begin{tabular}{|c|c|c|c|c|c|c|c|c|}
\hline \multirow{2}{*}{$\begin{array}{l}\text { Dependent variable } \\
\text { Hedonic happiness }\end{array}$} & \multicolumn{4}{|c|}{ Visibility } & \multicolumn{4}{|c|}{ API } \\
\hline & (1) & (2) & (3) & (4) & (5) & (6) & (7) & (8) \\
\hline \multirow[t]{2}{*}{ Air quality } & $0.107^{* * *}$ & $2.179^{* * *}$ & $2.010^{\star \star \star}$ & $1.879^{* * *}$ & $-0.090^{\star *}$ & -0.343 & $-1.621^{* *}$ & $-1.451^{\star *}$ \\
\hline & $(0.033)$ & $(0.660)$ & $(0.690)$ & $(0.678)$ & $(0.036)$ & $(0.682)$ & $(0.770)$ & $(0.727)$ \\
\hline \multirow[t]{2}{*}{ Sunshine } & & $2.063^{* \star *}$ & $2.537^{\star \star \star}$ & 0.629 & & -0.184 & -1.501 & $-3.136^{* *}$ \\
\hline & & $(0.546)$ & $(0.736)$ & $(1.034)$ & & $(1.223)$ & $(1.170)$ & $(1.269)$ \\
\hline \multirow[t]{2}{*}{ Air quality $\times$ Sunshine } & & $-0.839^{* * *}$ & $-0.765^{\star * *}$ & $-0.714^{* * *}$ & & 0.120 & $0.632^{* *}$ & $0.559^{*}$ \\
\hline & & $(0.261)$ & $(0.273)$ & $(0.270)$ & & $(0.271)$ & $(0.307)$ & $(0.291)$ \\
\hline \multirow[t]{2}{*}{ Household per capita income } & $0.055^{* * *}$ & $0.056^{* * *}$ & $0.055^{\star * *}$ & $0.055^{* * *}$ & $0.054^{* * *}$ & $0.054^{* * *}$ & $0.054^{* * *}$ & $0.054^{* * *}$ \\
\hline & $(0.010)$ & $(0.010)$ & $(0.010)$ & $(0.010)$ & $(0.010)$ & $(0.010)$ & $(0.010)$ & $(0.010)$ \\
\hline \multirow[t]{2}{*}{ Relative income } & $0.065^{* * *}$ & $0.066^{\star * *}$ & $0.066^{\star \star \star}$ & $0.066^{* * *}$ & $0.066^{* * *}$ & $0.066^{\star \star \star}$ & $0.066^{\star \star \star}$ & $0.066^{* * *}$ \\
\hline & $(0.009)$ & $(0.009)$ & $(0.009)$ & $(0.009)$ & $(0.009)$ & $(0.009)$ & $(0.009)$ & $(0.009)$ \\
\hline \multirow{2}{*}{ Age $(\div 10)$} & $-0.078^{* *}$ & $-0.078^{* *}$ & $-0.075^{\star *}$ & $-0.073^{* *}$ & $-0.080^{* *}$ & $-0.081^{* *}$ & $-0.080^{* *}$ & $-0.077^{* *}$ \\
\hline & $(0.037)$ & $(0.037)$ & $(0.037)$ & $(0.037)$ & $(0.037)$ & $(0.037)$ & $(0.037)$ & $(0.037)$ \\
\hline \multirow[t]{2}{*}{ Age $(\div 10)$ squared } & $0.007^{*}$ & $0.006^{*}$ & $0.006^{*}$ & $0.006^{\star}$ & $0.007^{*}$ & $0.007^{*}$ & $0.007^{\star}$ & $0.006^{*}$ \\
\hline & $(0.004)$ & $(0.004)$ & $(0.004)$ & $(0.004)$ & $(0.004)$ & $(0.004)$ & $(0.004)$ & $(0.004)$ \\
\hline \multirow[t]{2}{*}{ Male } & $0.071^{* * *}$ & $0.073^{* * *}$ & $0.074^{* * *}$ & $0.074^{* * *}$ & $0.071^{* * *}$ & $0.072^{* * *}$ & $0.073^{* * *}$ & $0.074^{* * *}$ \\
\hline & $(0.015)$ & $(0.015)$ & $(0.015)$ & $(0.015)$ & $(0.015)$ & $(0.015)$ & $(0.015)$ & $(0.015)$ \\
\hline \multirow[t]{2}{*}{ Married } & $0.108^{* * *}$ & $0.105^{\star * *}$ & $0.104^{\star \star *}$ & $0.102^{\star \star *}$ & $0.110^{* * *}$ & $0.109^{\star * \star}$ & $0.107^{\star \star \star *}$ & $0.105^{\star * *}$ \\
\hline & $(0.028)$ & $(0.028)$ & $(0.028)$ & $(0.028)$ & $(0.028)$ & $(0.028)$ & $(0.028)$ & $(0.028)$ \\
\hline \multirow[t]{2}{*}{ Divorced } & $-0.107^{*}$ & $-0.110^{*}$ & $-0.112^{*}$ & $-0.114^{*}$ & $-0.103^{*}$ & $-0.104^{*}$ & $-0.107^{*}$ & $-0.109^{*}$ \\
\hline & $(0.059)$ & $(0.059)$ & $(0.059)$ & $(0.059)$ & $(0.059)$ & $(0.059)$ & $(0.059)$ & $(0.059)$ \\
\hline \multirow[t]{2}{*}{ Education } & $0.015^{* * *}$ & $0.015^{\star * \star}$ & $0.014^{\star \star \star}$ & $0.014^{* * *}$ & $0.015^{\star * *}$ & $0.015^{\star \star \star}$ & $0.015^{\star * *}$ & $0.014^{* \star *}$ \\
\hline & $(0.003)$ & $(0.003)$ & $(0.003)$ & $(0.003)$ & $(0.003)$ & $(0.003)$ & $(0.003)$ & $(0.003)$ \\
\hline \multirow[t]{2}{*}{ Unemployed } & -0.085 & -0.091 & -0.095 & -0.098 & -0.089 & -0.084 & -0.094 & -0.098 \\
\hline & $(0.080)$ & $(0.080)$ & $(0.080)$ & $(0.080)$ & $(0.080)$ & $(0.081)$ & $(0.080)$ & $(0.080)$ \\
\hline \multirow[t]{2}{*}{ State employee } & -0.035 & -0.034 & -0.032 & -0.034 & -0.036 & -0.034 & -0.031 & -0.032 \\
\hline & $(0.027)$ & $(0.027)$ & $(0.027)$ & $(0.027)$ & $(0.027)$ & $(0.027)$ & $(0.027)$ & $(0.027)$ \\
\hline \multirow[t]{2}{*}{ Party } & $0.113^{* * *}$ & $0.113^{* * *}$ & $0.114^{* * *}$ & $0.113^{* * *}$ & $0.112^{* * *}$ & $0.112^{* * *}$ & $0.113^{* * *}$ & $0.112^{* * *}$ \\
\hline & $(0.031)$ & $(0.031)$ & $(0.031)$ & $(0.031)$ & $(0.031)$ & $(0.031)$ & $(0.031)$ & $(0.031)$ \\
\hline \multirow[t]{2}{*}{ Chronic disease } & $-0.122^{* * *}$ & $-0.125^{* * *}$ & $-0.126^{* \star *}$ & $-0.125^{\star \star \star}$ & $-0.121^{* * *}$ & $-0.122^{* \star *}$ & $-0.123^{* * *}$ & $-0.123^{* * *}$ \\
\hline & $(0.027)$ & $(0.027)$ & $(0.027)$ & $(0.027)$ & $(0.027)$ & $(0.027)$ & $(0.027)$ & $(0.027)$ \\
\hline County fixed effect & Yes & Yes & Yes & Yes & Yes & Yes & Yes & Yes \\
\hline Weather controls & No & No & Yes & Yes & No & No & Yes & Yes \\
\hline Month, day-of-week fixed effects & No & No & No & Yes & No & No & No & Yes \\
\hline Observations & 13,682 & 13,682 & 13,682 & 13,682 & 13,736 & 13,736 & 13,736 & 13,736 \\
\hline$R$-squared & .143 & .146 & .147 & .149 & .142 & .143 & .145 & .146 \\
\hline
\end{tabular}

Source: China Family Panel Studies (ISSS 2012).

Note: API = air pollution index. The weather controls include mean temperature and its square, temperature difference (daily maximum-minimum), total precipitation, maximum sustained wind speed, and a dummy for bad weather. Robust standard errors, clustered at the county level, are presented in parentheses. $* 10 \%$ significance level. $* * 5 \%$ significance level. $* * * 1 \%$ significance level. 
Table 4.3 Effect of air quality (visibility versus API) on mental well-being, longitudinal

\begin{tabular}{|c|c|c|c|c|c|c|c|c|}
\hline \multirow{2}{*}{$\begin{array}{l}\text { Dependent variable } \\
\text { Percentile of the CES-D score }\end{array}$} & \multicolumn{4}{|c|}{ Visibility } & \multicolumn{4}{|c|}{ API } \\
\hline & $(1)$ & $(2)$ & (3) & $(4)$ & (5) & (6) & $(7)$ & (8) \\
\hline Air quality & $\begin{array}{l}-0.007 \\
(0.010)\end{array}$ & $\begin{array}{l}-0.578^{*} \\
(0.296)\end{array}$ & $\begin{array}{l}-0.613^{* *} \\
(0.266)\end{array}$ & $\begin{array}{l}-0.546^{* *} \\
(0.263)\end{array}$ & $\begin{array}{l}-0.001 \\
(0.012)\end{array}$ & $\begin{array}{l}0.880^{\star *} \\
(0.366)\end{array}$ & $\begin{array}{l}0.991^{* *} \\
(0.460)\end{array}$ & $\begin{array}{l}1.058^{* *} \\
(0.461)\end{array}$ \\
\hline Sunshine & & $\begin{array}{l}-0.335 \\
(0.223)\end{array}$ & $\begin{array}{l}-0.187 \\
(0.230)\end{array}$ & $\begin{array}{c}0.181 \\
(0.333)\end{array}$ & & $\begin{array}{l}1.576^{* *} \\
(0.643)\end{array}$ & $\begin{array}{l}1.814^{* *} \\
(0.713)\end{array}$ & $\begin{array}{c}2.313^{* * *} \\
(0.776)\end{array}$ \\
\hline Air quality $\times$ Sunshine & & $\begin{array}{l}0.225^{\star} \\
(0.116)\end{array}$ & $\begin{array}{l}0.239^{* *} \\
(0.105)\end{array}$ & $\begin{array}{l}0.215^{\star *} \\
(0.104)\end{array}$ & & $\begin{array}{c}-0.342^{* *} \\
(0.145)\end{array}$ & $\begin{array}{c}-0.385^{* *} \\
(0.181)\end{array}$ & $\begin{array}{c}-0.416^{* *} \\
(0.181)\end{array}$ \\
\hline Household per capita income & $\begin{array}{l}-0.000 \\
(0.004)\end{array}$ & $\begin{array}{l}-0.001 \\
(0.004)\end{array}$ & $\begin{array}{l}-0.000 \\
(0.004)\end{array}$ & $\begin{array}{c}0.000 \\
(0.004)\end{array}$ & $\begin{array}{c}0.001 \\
(0.004)\end{array}$ & $\begin{array}{l}0.002 \\
(0.004)\end{array}$ & $\begin{array}{c}0.002 \\
(0.004)\end{array}$ & $\begin{array}{l}0.002 \\
(0.004)\end{array}$ \\
\hline Relative income & $\begin{array}{c}-0.019^{* * *} \\
(0.003)\end{array}$ & $\begin{array}{c}-0.019^{* * *} \\
(0.003)\end{array}$ & $\begin{array}{c}-0.018^{* * *} \\
(0.003)\end{array}$ & $\begin{array}{l}-0.018^{* * *} \\
(0.003)\end{array}$ & $\begin{array}{l}-0.019^{* * *} \\
(0.004)\end{array}$ & $\begin{array}{c}-0.019^{* * *} \\
(0.004)\end{array}$ & $\begin{array}{l}-0.018^{* * *} \\
(0.004)\end{array}$ & $\begin{array}{c}-0.018^{* * *} \\
(0.004)\end{array}$ \\
\hline Age $(\div 10)$ squared & $\begin{array}{c}0.001 \\
(0.004)\end{array}$ & $\begin{array}{l}0.005 \\
(0.004)\end{array}$ & $\begin{array}{l}0.009^{* *} \\
(0.005)\end{array}$ & $\begin{array}{c}0.035^{* * *} \\
(0.008)\end{array}$ & $\begin{array}{l}-0.000 \\
(0.005)\end{array}$ & $\begin{array}{l}0.006 \\
(0.005)\end{array}$ & $\begin{array}{l}0.009^{*} \\
(0.005)\end{array}$ & $\begin{array}{l}0.031^{* * *} \\
(0.009)\end{array}$ \\
\hline Married & $\begin{array}{c}-0.094^{* * *} \\
(0.017)\end{array}$ & $\begin{array}{c}-0.092^{* * *} \\
(0.017)\end{array}$ & $\begin{array}{c}-0.089^{* * *} \\
(0.017)\end{array}$ & $\begin{array}{c}-0.072^{* * *} \\
(0.016)\end{array}$ & $\begin{array}{c}-0.087^{* * *} \\
(0.018)\end{array}$ & $\begin{array}{c}-0.080^{* * *} \\
(0.017)\end{array}$ & $\begin{array}{c}-0.078^{* * *} \\
(0.017)\end{array}$ & $\begin{array}{c}-0.063^{* * *} \\
(0.018)\end{array}$ \\
\hline Divorced & $\begin{array}{l}-0.004 \\
(0.038)\end{array}$ & $\begin{array}{c}0.001 \\
(0.038)\end{array}$ & $\begin{array}{c}0.005 \\
(0.037)\end{array}$ & $\begin{array}{c}0.025 \\
(0.038)\end{array}$ & $\begin{array}{l}-0.020 \\
(0.040)\end{array}$ & $\begin{array}{l}-0.012 \\
(0.039)\end{array}$ & $\begin{array}{l}-0.009 \\
(0.039)\end{array}$ & $\begin{array}{l}0.009 \\
(0.041)\end{array}$ \\
\hline Education & $\begin{array}{l}-0.000 \\
(0.002)\end{array}$ & $\begin{array}{l}-0.000 \\
(0.002)\end{array}$ & $\begin{array}{l}-0.000 \\
(0.002)\end{array}$ & $\begin{array}{c}0.000 \\
(0.002)\end{array}$ & $\begin{array}{l}-0.002 \\
(0.002)\end{array}$ & $\begin{array}{l}-0.002 \\
(0.002)\end{array}$ & $\begin{array}{l}-0.002 \\
(0.002)\end{array}$ & $\begin{array}{l}-0.002 \\
(0.002)\end{array}$ \\
\hline Unemployed & $\begin{array}{l}0.043^{* * *} \\
(0.014)\end{array}$ & $\begin{array}{c}0.037^{\star \star *} \\
(0.014)\end{array}$ & $\begin{array}{l}0.037^{* *} \\
(0.014)\end{array}$ & $\begin{array}{l}0.028^{\star \star} \\
(0.014)\end{array}$ & $\begin{array}{c}0.046^{* * *} \\
(0.016)\end{array}$ & $\begin{array}{c}0.043^{* * *} \\
(0.015)\end{array}$ & $\begin{array}{c}0.043^{* * *} \\
(0.015)\end{array}$ & $\begin{array}{l}0.035^{\star *} \\
(0.015)\end{array}$ \\
\hline State employee & $\begin{array}{l}-0.003 \\
(0.012)\end{array}$ & $\begin{array}{l}-0.003 \\
(0.012)\end{array}$ & $\begin{array}{l}-0.002 \\
(0.012)\end{array}$ & $\begin{array}{l}-0.000 \\
(0.012)\end{array}$ & $\begin{array}{l}-0.002 \\
(0.013)\end{array}$ & $\begin{array}{c}-0.001 \\
(0.013)\end{array}$ & $\begin{array}{l}-0.001 \\
(0.013)\end{array}$ & $\begin{array}{c}0.002 \\
(0.013)\end{array}$ \\
\hline Party & $\begin{array}{l}-0.023 \\
(0.021)\end{array}$ & $\begin{array}{l}-0.022 \\
(0.020)\end{array}$ & $\begin{array}{l}-0.023 \\
(0.020)\end{array}$ & $\begin{array}{l}-0.018 \\
(0.020)\end{array}$ & $\begin{array}{l}-0.004 \\
(0.024)\end{array}$ & $\begin{array}{l}-0.006 \\
(0.024)\end{array}$ & $\begin{array}{l}-0.004 \\
(0.023)\end{array}$ & $\begin{array}{l}-0.001 \\
(0.023)\end{array}$ \\
\hline Chronic disease & $\begin{array}{l}0.045^{* * *} \\
(0.008)\end{array}$ & $\begin{array}{c}0.044^{\star \star *} \\
(0.008)\end{array}$ & $\begin{array}{c}0.044^{\star * *} \\
(0.008)\end{array}$ & $\begin{array}{c}0.044^{* * *} \\
(0.008)\end{array}$ & $\begin{array}{c}0.038^{* * *} \\
(0.009)\end{array}$ & $\begin{array}{c}0.037^{\star * *} \\
(0.009)\end{array}$ & $\begin{array}{c}0.037^{\star * *} \\
(0.009)\end{array}$ & $\begin{array}{l}0.037^{\star * *} \\
(0.009)\end{array}$ \\
\hline Individual fixed effect & Yes & Yes & Yes & Yes & Yes & Yes & Yes & Yes \\
\hline Weather controls & No & No & Yes & Yes & No & No & Yes & Yes \\
\hline Year, month, day-of-week fixed effects & No & No & No & Yes & No & No & No & Yes \\
\hline $\begin{array}{l}\text { Observations } \\
\text { (Within) } R \text {-squared }\end{array}$ & $\begin{array}{c}27,070 \\
.013\end{array}$ & $\begin{array}{c}27,070 \\
.019\end{array}$ & $\begin{array}{c}27,070 \\
.022\end{array}$ & $\begin{array}{c}27,070 \\
.030\end{array}$ & $\begin{array}{c}24,398 \\
.011\end{array}$ & $\begin{array}{c}24,398 \\
.017\end{array}$ & $\begin{array}{c}24,398 \\
.020\end{array}$ & $\begin{array}{c}24,398 \\
.029\end{array}$ \\
\hline
\end{tabular}

Source: China Family Panel Studies (ISSS 2010; 2012).

Note: API = air pollution index; CES-D = Center for Epidemiologic Studies Depression scale. Lower percentile of the CES-D score indicates better mental well-being. The weather controls include mean temperature and its square, temperature difference (daily maximum-minimum), total precipitation, maximum sustained wind speed, and a dummy for bad weather. Robust standard errors, clustered at the county level, are presented in parentheses. * $10 \%$ significance level. **5\% significance level. *** $1 \%$ significance level. 
Table 4.4 and Table 4.5 repeat the estimations with two binary measures of mental well-being, namely, depressive symptoms (with CES-D score greater than 15) and severe depressive symptoms (with CES-D score greater than 20). Since there are no depressive symptom cut-offs for the six-item CES-D, we have to rely on cross-sectional estimations. As shown in columns (2) through (4) of Table 4.4 and Table 4.5, after controlling for demographic characteristics of the respondents, low visibility on the day of interview is positively correlated with the chances of depressive symptoms and severe depression. In contrast, API does not seem to have any significant effect.

If contemporary exposure to air pollution is really the driving force of the observed decline in hedonic happiness and mental well-being, we should observe no such effect when the timing of air pollution and the date of interview are mismatched. Results from the placebo test that forwards air visibility by a week (Table A.3) indeed suggest that no unobserved factors drive the observed pattern between air quality and SWB.

Life satisfaction is largely immune to short-term variations in air pollution (Table 4.1), so we further investigate to what extent they are susceptible to deviation of pollution from various time trends, such as a week, a month, a season, and a year. One would hypothesize that a greater deviation from these pollution trends may generate a larger impact on life satisfaction. Results in Table 4.6 (columns [1] through [4] for visibility and columns [5] through [8] for API), however, suggest that life satisfaction is not sensitive to deviations from these trends.

The muted impact on life satisfaction probably can be explained by hedonic adaptation, processes that attenuate the long-term emotional impact of unfavorable circumstances. Hedonic adaptation is often evolutionarily optimal in protecting people from adverse mental reactions, preventing the continued expenditure of energy on futile attempts to change the unchangeable and redirecting motivation to changes that can be made (Frederick and Loewenstein 1999). For example, the level of air pollution that was previously very hedonically negative may become hedonically neutral over time as people transition the neutral reference point from lower to higher levels of pollution. 
Table 4.4 Effect of air quality (visibility versus API) on depressive symptoms, cross-sectional

\begin{tabular}{|c|c|c|c|c|c|c|c|c|}
\hline \multirow{2}{*}{$\begin{array}{l}\text { Dependent variable } \\
\text { Depressive symptoms }\end{array}$} & \multicolumn{4}{|c|}{ Visibility } & \multicolumn{4}{|c|}{ API } \\
\hline & (1) & $(2)$ & (3) & (4) & (5) & (6) & (7) & (8) \\
\hline \multirow[t]{2}{*}{ Air quality } & -0.023 & $-0.733^{\star * *}$ & $-0.807^{* * *}$ & $-0.750^{\star * *}$ & -0.007 & 0.423 & 0.402 & 0.297 \\
\hline & $(0.016)$ & $(0.248)$ & $(0.264)$ & $(0.282)$ & $(0.017)$ & $(0.472)$ & $(0.477)$ & $(0.492)$ \\
\hline \multirow[t]{2}{*}{ Sunshine } & & $-0.461^{* *}$ & -0.502 & -0.072 & & 0.788 & 0.642 & 1.261 \\
\hline & & $(0.216)$ & $(0.354)$ & $(0.497)$ & & $(0.790)$ & $(0.804)$ & $(0.812)$ \\
\hline \multirow[t]{2}{*}{ Air quality×Sunshine } & & $0.283^{* * *}$ & $0.313^{* * *}$ & $0.291^{\star \star}$ & & -0.164 & -0.157 & -0.115 \\
\hline & & $(0.099)$ & $(0.105)$ & $(0.112)$ & & $(0.186)$ & $(0.188)$ & $(0.194)$ \\
\hline \multirow[t]{2}{*}{ Household per capita income } & $-0.023^{* * *}$ & $-0.023^{* * *}$ & $-0.023^{* * *}$ & $-0.023^{* * *}$ & $-0.024^{* * *}$ & $-0.023^{* * *}$ & $-0.023^{* * *}$ & $-0.023^{* * *}$ \\
\hline & $(0.005)$ & $(0.005)$ & $(0.005)$ & $(0.005)$ & $(0.005)$ & $(0.005)$ & $(0.005)$ & $(0.005)$ \\
\hline \multirow[t]{2}{*}{ Relative income } & $-0.037^{* * *}$ & $-0.037^{* * *}$ & $-0.037^{* * *}$ & $-0.037^{* * *}$ & $-0.037^{* * *}$ & $-0.037^{* * *}$ & $-0.037^{* * *}$ & $-0.037^{* * *}$ \\
\hline & $(0.005)$ & $(0.005)$ & $(0.005)$ & $(0.005)$ & $(0.005)$ & $(0.005)$ & $(0.005)$ & $(0.005)$ \\
\hline \multirow[t]{2}{*}{ Age $(\div 10)$} & $0.059^{\star \star \star}$ & $0.059^{* * *}$ & $0.059^{* * *}$ & $0.058^{* * *}$ & $0.060^{* * *}$ & $0.060^{* * *}$ & $0.061^{* * *}$ & $0.060^{* * *}$ \\
\hline & $(0.017)$ & $(0.017)$ & $(0.017)$ & $(0.017)$ & $(0.017)$ & $(0.017)$ & $(0.017)$ & $(0.017)$ \\
\hline \multirow[t]{2}{*}{ Age $(\div 10)$ squared } & $-0.005^{* * *}$ & $-0.005^{\star * *}$ & $-0.005^{* * *}$ & $-0.005^{\star * *}$ & $-0.005^{\star * *}$ & $-0.005^{* * *}$ & $-0.005^{* * *}$ & $-0.005^{* * *}$ \\
\hline & $(0.002)$ & $(0.002)$ & $(0.002)$ & $(0.002)$ & $(0.002)$ & $(0.002)$ & $(0.002)$ & $(0.002)$ \\
\hline \multirow[t]{2}{*}{ Male } & $-0.079^{* * *}$ & $-0.079^{* * *}$ & $-0.079^{* * *}$ & $-0.079^{* * *}$ & $-0.078^{* * *}$ & $-0.078^{* * *}$ & $-0.078^{* * *}$ & $-0.078^{* * *}$ \\
\hline & $(0.008)$ & $(0.008)$ & $(0.008)$ & $(0.008)$ & $(0.008)$ & $(0.008)$ & $(0.008)$ & $(0.008)$ \\
\hline \multirow[t]{2}{*}{ Married } & $-0.115^{\star * *}$ & $-0.115^{\star * *}$ & $-0.115^{\star * *}$ & $-0.114^{* * *}$ & $-0.114^{* * *}$ & $-0.114^{* * *}$ & $-0.115^{\star * *}$ & $-0.114^{* * *}$ \\
\hline & $(0.014)$ & $(0.014)$ & $(0.014)$ & $(0.014)$ & $(0.014)$ & $(0.014)$ & $(0.014)$ & $(0.014)$ \\
\hline \multirow[t]{2}{*}{ Divorced } & 0.042 & 0.043 & 0.043 & 0.043 & 0.041 & 0.041 & 0.042 & 0.042 \\
\hline & $(0.034)$ & $(0.034)$ & $(0.034)$ & $(0.034)$ & $(0.034)$ & $(0.034)$ & $(0.034)$ & $(0.034)$ \\
\hline \multirow[t]{2}{*}{ Education } & $-0.009^{* * *}$ & $-0.009^{\star \star *}$ & $-0.009^{* * *}$ & $-0.009^{\star \star *}$ & $-0.010^{\star \star \star}$ & $-0.010^{\star * *}$ & $-0.010^{\star \star \star}$ & $-0.009^{* * *}$ \\
\hline & $(0.001)$ & $(0.001)$ & $(0.001)$ & $(0.001)$ & $(0.001)$ & $(0.001)$ & $(0.001)$ & $(0.001)$ \\
\hline \multirow[t]{2}{*}{ Unemployed } & $0.101^{\star * *}$ & $0.106^{* * *}$ & $0.106^{\star \star *}$ & $0.105^{\star * *}$ & $0.100^{* * *}$ & $0.102^{\star \star \star}$ & $0.102^{* * *}$ & $0.103^{* \star *}$ \\
\hline & $(0.033)$ & $(0.032)$ & $(0.032)$ & $(0.033)$ & $(0.034)$ & $(0.033)$ & $(0.033)$ & $(0.033)$ \\
\hline \multirow[t]{2}{*}{ State employee } & -0.006 & -0.006 & -0.006 & -0.005 & -0.006 & -0.006 & -0.006 & -0.005 \\
\hline & $(0.012)$ & $(0.012)$ & $(0.012)$ & $(0.012)$ & $(0.012)$ & $(0.012)$ & $(0.012)$ & $(0.012)$ \\
\hline \multirow[t]{2}{*}{ Party } & $-0.039^{* * *}$ & $-0.039^{* * *}$ & $-0.039^{* * *}$ & $-0.039^{* * *}$ & $-0.037^{* * *}$ & $-0.037^{* * *}$ & $-0.037^{* * *}$ & $-0.037^{* * *}$ \\
\hline & $(0.012)$ & $(0.012)$ & $(0.012)$ & $(0.012)$ & $(0.012)$ & $(0.012)$ & $(0.012)$ & $(0.012)$ \\
\hline \multirow[t]{2}{*}{ Chronic disease } & $0.115^{\star * *}$ & $0.114^{* * *}$ & $0.114^{* * *}$ & $0.115^{\star * *}$ & $0.113^{* * *}$ & $0.113^{* * *}$ & $0.113^{* * *}$ & $0.114^{* * *}$ \\
\hline & $(0.012)$ & $(0.012)$ & $(0.012)$ & $(0.012)$ & $(0.012)$ & $(0.012)$ & $(0.012)$ & $(0.012)$ \\
\hline County fixed effect & Yes & Yes & Yes & Yes & Yes & Yes & Yes & Yes \\
\hline Weather controls & No & No & Yes & Yes & No & No & Yes & Yes \\
\hline Month, day-of-week fixed effects & No & No & No & Yes & No & No & No & Yes \\
\hline Observations & 13,433 & 13,433 & 13,433 & 13,433 & 13,483 & 13,483 & 13,483 & 13,483 \\
\hline$R$-squared & .155 & .156 & .157 & .157 & .155 & .155 & .156 & .157 \\
\hline
\end{tabular}

Source: China Family Panel Studies (ISSS 2012).

Note: API = air pollution index. The weather controls include mean temperature and its square, temperature difference (daily maximum-minimum), total precipitation,

maximum sustained wind speed, and a dummy for bad weather. Robust standard errors, clustered at the county level, are presented in parentheses. $* * 5 \%$ significance level. ***1\% significance level. 
Table 4.5 Effect of air quality (visibility versus API) on severe depression, cross-sectional

\begin{tabular}{|c|c|c|c|c|c|c|c|c|}
\hline \multirow{2}{*}{$\begin{array}{l}\text { Dependent variable } \\
\text { Severe depression }\end{array}$} & \multicolumn{4}{|c|}{ Visibility } & \multicolumn{4}{|c|}{ API } \\
\hline & (1) & $(2)$ & (3) & (4) & (5) & (6) & (7) & (8) \\
\hline \multirow[t]{2}{*}{ Air quality } & 0.005 & $-0.344^{* *}$ & $-0.376^{* *}$ & $-0.330^{* *}$ & -0.019 & 0.249 & 0.325 & 0.255 \\
\hline & $(0.012)$ & $(0.143)$ & $(0.145)$ & $(0.145)$ & $(0.012)$ & $(0.258)$ & $(0.284)$ & $(0.293)$ \\
\hline \multirow[t]{2}{*}{ Sunshine } & & -0.145 & -0.210 & -0.025 & & 0.540 & 0.523 & 0.771 \\
\hline & & $(0.133)$ & $(0.213)$ & $(0.321)$ & & $(0.434)$ & $(0.461)$ & $(0.472)$ \\
\hline \multirow[t]{2}{*}{ Air quality $\times$ Sunshine } & & $0.137^{* *}$ & $0.149^{* *}$ & $0.131^{* *}$ & & -0.099 & -0.130 & -0.101 \\
\hline & & $(0.059)$ & $(0.060)$ & $(0.060)$ & & $(0.102)$ & $(0.113)$ & $(0.117)$ \\
\hline \multirow[t]{2}{*}{ Household per capita income } & $-0.015^{\star * *}$ & $-0.015^{* * *}$ & $-0.015^{* * *}$ & $-0.015^{* * *}$ & $-0.016^{* * *}$ & $-0.015^{* * *}$ & $-0.015^{* * *}$ & $-0.015^{* * *}$ \\
\hline & $(0.003)$ & $(0.003)$ & $(0.003)$ & $(0.003)$ & $(0.003)$ & $(0.003)$ & $(0.003)$ & $(0.003)$ \\
\hline \multirow[t]{2}{*}{ Relative income } & $-0.027^{* * *}$ & $-0.027^{* * *}$ & $-0.027^{* * *}$ & $-0.027^{\star * *}$ & $-0.027^{\star \star \star}$ & $-0.027^{\star \star *}$ & $-0.027^{\star \star \star}$ & $-0.027^{* * *}$ \\
\hline & $(0.004)$ & $(0.004)$ & $(0.004)$ & $(0.004)$ & $(0.004)$ & $(0.004)$ & $(0.004)$ & $(0.004)$ \\
\hline \multirow{2}{*}{ Age $(\div 10)$} & $0.056^{* * *}$ & $0.055^{* * *}$ & $0.055^{* * *}$ & $0.054^{* * *}$ & $0.056^{* * *}$ & $0.056^{* * *}$ & $0.056^{* * *}$ & $0.055^{* * *}$ \\
\hline & $(0.012)$ & $(0.012)$ & $(0.012)$ & $(0.012)$ & $(0.012)$ & $(0.012)$ & $(0.012)$ & $(0.012)$ \\
\hline \multirow[t]{2}{*}{ Age $(\div 10)$ squared } & $-0.004^{* * *}$ & $-0.004^{* * *}$ & $-0.004^{* * *}$ & $-0.004^{* * *}$ & $-0.004^{* * *}$ & $-0.004^{* * *}$ & $-0.004^{* * *}$ & $-0.004^{* * *}$ \\
\hline & $(0.001)$ & $(0.001)$ & $(0.001)$ & $(0.001)$ & $(0.001)$ & $(0.001)$ & $(0.001)$ & $(0.001)$ \\
\hline \multirow[t]{2}{*}{ Male } & $-0.055^{* * *}$ & $-0.055^{\star * *}$ & $-0.055^{* * *}$ & $-0.055^{* * *}$ & $-0.055^{\star * *}$ & $-0.054^{* * *}$ & $-0.054^{* * *}$ & $-0.054^{* * *}$ \\
\hline & $(0.006)$ & $(0.006)$ & $(0.006)$ & $(0.006)$ & $(0.006)$ & $(0.006)$ & $(0.006)$ & $(0.006)$ \\
\hline \multirow[t]{2}{*}{ Married } & $-0.093^{* * *}$ & $-0.094^{* * *}$ & $-0.094^{* * *}$ & $-0.093^{* * *}$ & $-0.093^{* * *}$ & $-0.093^{* * *}$ & $-0.093^{* * *}$ & $-0.092^{* * *}$ \\
\hline & $(0.011)$ & $(0.011)$ & $(0.011)$ & $(0.011)$ & $(0.011)$ & $(0.011)$ & $(0.011)$ & $(0.011)$ \\
\hline \multirow[t]{2}{*}{ Divorced } & -0.008 & -0.007 & -0.007 & -0.007 & -0.007 & -0.007 & -0.007 & -0.007 \\
\hline & $(0.025)$ & $(0.024)$ & $(0.024)$ & $(0.024)$ & $(0.025)$ & $(0.024)$ & $(0.024)$ & $(0.024)$ \\
\hline \multirow[t]{2}{*}{ Education } & $-0.006^{\star * *}$ & $-0.006^{* * *}$ & $-0.006^{* * *}$ & $-0.006^{* * *}$ & $-0.006^{\star * *}$ & $-0.006^{* * *}$ & $-0.006^{* * *}$ & $-0.006^{* * *}$ \\
\hline & $(0.001)$ & $(0.001)$ & $(0.001)$ & $(0.001)$ & $(0.001)$ & $(0.001)$ & $(0.001)$ & $(0.001)$ \\
\hline \multirow[t]{2}{*}{ Unemployed } & -0.002 & 0.001 & 0.001 & 0.001 & -0.003 & -0.001 & -0.001 & 0.000 \\
\hline & $(0.023)$ & $(0.023)$ & $(0.023)$ & $(0.024)$ & $(0.023)$ & $(0.023)$ & $(0.023)$ & $(0.024)$ \\
\hline \multirow[t]{2}{*}{ State employee } & 0.011 & 0.012 & 0.012 & 0.012 & 0.011 & 0.012 & 0.012 & 0.012 \\
\hline & $(0.010)$ & $(0.010)$ & $(0.010)$ & $(0.010)$ & $(0.010)$ & $(0.010)$ & $(0.010)$ & $(0.010)$ \\
\hline \multirow[t]{2}{*}{ Party } & $-0.031^{* * *}$ & $-0.031^{* * *}$ & $-0.031^{* * *}$ & $-0.031^{* * *}$ & $-0.030^{* * *}$ & $-0.029^{* * *}$ & $-0.029^{* * *}$ & $-0.029^{* * *}$ \\
\hline & $(0.009)$ & $(0.009)$ & $(0.009)$ & $(0.009)$ & $(0.009)$ & $(0.009)$ & $(0.009)$ & $(0.009)$ \\
\hline \multirow[t]{2}{*}{ Chronic disease } & $0.102^{* * *}$ & $0.101^{* * *}$ & $0.101^{* * *}$ & $0.101^{* * *}$ & $0.100^{* * *}$ & $0.100^{* * *}$ & $0.100^{* * *}$ & $0.100^{* * *}$ \\
\hline & $(0.011)$ & $(0.011)$ & $(0.011)$ & $(0.011)$ & $(0.011)$ & $(0.011)$ & $(0.011)$ & $(0.011)$ \\
\hline County fixed effect & Yes & Yes & Yes & Yes & Yes & Yes & Yes & Yes \\
\hline Weather controls & No & No & Yes & Yes & No & No & Yes & Yes \\
\hline Month, day-of-week fixed effects & No & No & No & Yes & No & No & No & Yes \\
\hline Observations & 13,433 & 13,433 & 13,433 & 13,433 & 13,483 & 13,483 & 13,483 & 13,483 \\
\hline$R$-squared & .121 & .123 & .123 & .125 & .121 & .122 & .122 & .124 \\
\hline
\end{tabular}

Source: China Family Panel Studies (ISSS 2012).

Note: API = air pollution index. The weather controls include mean temperature and its square, temperature difference (daily maximum-minimum), total precipitation,

maximum sustained wind speed, and a dummy for bad weather. Robust standard errors, clustered at the county level, are presented in parentheses. $* * 5 \%$ significance level. ***1\% significance level. 
Table 4.6 Deviation-from-the-mean effect of air quality on life satisfaction

\begin{tabular}{|c|c|c|c|c|}
\hline \multicolumn{5}{|c|}{ A. Visibility } \\
\hline Dependent variable & 7-day deviation & $\begin{array}{c}\text { 30-day } \\
\text { deviation }\end{array}$ & $\begin{array}{c}\text { 90-day } \\
\text { deviation }\end{array}$ & $\begin{array}{c}\text { 365-day } \\
\text { deviation }\end{array}$ \\
\hline Life satisfaction & (1) & (2) & (3) & (4) \\
\hline $1 \nabla^{k-1}$ & 0.000 & 0.005 & 0.022 & 0.016 \\
\hline $\ln P_{t}-\bar{k} \sum_{i=0} \ln P_{t-i}$ & $(0.031)$ & $(0.030)$ & $(0.032)$ & $(0.036)$ \\
\hline Per capita income & $\begin{array}{l}0.022^{*} \\
(0.012)\end{array}$ & $\begin{array}{l}0.022^{*} \\
(0.012)\end{array}$ & $\begin{array}{c}0.022^{*} \\
(0.012)\end{array}$ & $\begin{array}{c}0.022^{*} \\
(0.012)\end{array}$ \\
\hline Relative income & $0.204^{* * *}$ & $0.205^{* * *}$ & $0.206^{* * *}$ & $0.205^{\star * *}$ \\
\hline & $(0.012)$ & $(0.012)$ & $(0.012)$ & $(0.012)$ \\
\hline Observations & 27,408 & 27,392 & 27,332 & 26,674 \\
\hline$R$-squared & .054 & .054 & .054 & .053 \\
\hline \multicolumn{5}{|c|}{ B. API } \\
\hline Dependent variable & 7-day deviation & $\begin{array}{c}\text { 30-day } \\
\text { deviation }\end{array}$ & $\begin{array}{c}\text { 90-day } \\
\text { deviation }\end{array}$ & $\begin{array}{l}\text { 365-day } \\
\text { deviation }\end{array}$ \\
\hline Life satisfaction & (5) & (6) & (7) & $(8)$ \\
\hline $1 \nabla^{k-1}$ & -0.015 & -0.016 & -0.017 & -0.015 \\
\hline $\ln P_{t}-\bar{k} \sum_{i=0} \ln P_{t-i}$ & $(0.050)$ & $(0.051)$ & $(0.051)$ & $(0.051)$ \\
\hline Per capita income & $\begin{array}{l}0.026^{*} \\
(0.013)\end{array}$ & $\begin{array}{l}0.026^{*} \\
(0.013)\end{array}$ & $\begin{array}{l}0.026^{*} \\
(0.013)\end{array}$ & $\begin{array}{l}0.026^{*} \\
(0.013)\end{array}$ \\
\hline Relative income & $\begin{array}{c}0.203^{* * *} \\
(0.013)\end{array}$ & $\begin{array}{c}0.203^{* * *} \\
(0.013)\end{array}$ & $\begin{array}{c}0.203^{* * *} \\
(0.013)\end{array}$ & $\begin{array}{c}0.203^{* \star *} \\
(0.013)\end{array}$ \\
\hline Observations & 24,729 & 24,729 & 24,729 & 24,699 \\
\hline$R$-squared & .058 & .058 & .058 & .058 \\
\hline
\end{tabular}

Source: China Family Panel Studies (ISSS 2010; 2012).

Note: $\quad$ API $=$ air pollution index. $\frac{1}{k} \sum_{i=0}^{k-1} \ln P_{t-i}$ indicates the mean of the pollution level (visibility/API) in the past k days, where $\mathrm{k}$ equals $7,30,90$, and 365 , respectively. Other covariates and fixed effects are the same as those in column (4) of Table 4.1. Robust standard errors, clustered at the county level, are presented in parentheses. *10\% significance level. $* * * 1 \%$ significance level.

\section{Heterogeneity of the Effect}

Our baseline results suggest that the main effect of air quality is on hedonic happiness and mental wellbeing. This section further investigates heterogeneous effects, which may help us understand the potential pathways by which air quality affects SWB.

Table 4.7 presents results of responses to air quality by gender and age, respectively. Apparently females respond more strongly to air pollution than do males, while the elderly are more sensitive to air quality than are other age cohorts. This is probably because senior people spend more time outdoors and therefore care more about air quality. Table A.1 further shows the impact on men and women by age. The impact is particularly strong for older women. 
Table 4.7 Heterogeneity test of air quality effect, by gender and age

\begin{tabular}{|c|c|c|c|c|c|}
\hline \multicolumn{6}{|c|}{ A. Hedonic happiness } \\
\hline Dependent variable & \multicolumn{2}{|c|}{ Gender } & \multicolumn{3}{|c|}{ Age } \\
\hline \multirow[t]{2}{*}{ Hedonic happiness } & Male & Female & $\begin{array}{l}\text { Young } \\
(16-39)\end{array}$ & $\begin{array}{l}\text { Middle } \\
(40-59)\end{array}$ & $\begin{array}{l}\text { Old } \\
\text { (60 and } \\
\text { older) }\end{array}$ \\
\hline & (1) & (2) & (3) & (4) & $(5)$ \\
\hline $\begin{array}{l}\text { Dependent variable } \\
\text { mean }\end{array}$ & 2.911 & 2.796 & 2.918 & 2.830 & 2.830 \\
\hline Visibility & $\begin{array}{c}1.353 \\
(0.823)\end{array}$ & $\begin{array}{l}2.303^{* * *} \\
(0.665)\end{array}$ & $\begin{array}{l}1.378^{*} \\
(0.826)\end{array}$ & $\begin{array}{l}1.875^{\star *} \\
(0.919)\end{array}$ & $\begin{array}{c}1.935^{\star} \\
(1.125)\end{array}$ \\
\hline Sunshine & $\begin{array}{l}-0.285 \\
(1.233)\end{array}$ & $\begin{array}{c}1.381 \\
(1.117)\end{array}$ & $\begin{array}{l}-0.056 \\
(1.241)\end{array}$ & $\begin{array}{c}0.880 \\
(1.442)\end{array}$ & $\begin{array}{l}-0.632 \\
(1.834)\end{array}$ \\
\hline Visibility $\times$ Sunshine & $\begin{array}{l}-0.517 \\
(0.329)\end{array}$ & $\begin{array}{c}-0.875^{\star * *} \\
(0.268)\end{array}$ & $\begin{array}{l}-0.489 \\
(0.335)\end{array}$ & $\begin{array}{c}-0.713^{*} \\
(0.366)\end{array}$ & $\begin{array}{l}-0.737 \\
(0.445)\end{array}$ \\
\hline Per capita income & $\begin{array}{c}0.049^{* * *} \\
(0.014)\end{array}$ & $\begin{array}{c}0.062^{* * *} \\
(0.013)\end{array}$ & $\begin{array}{c}0.013 \\
(0.016)\end{array}$ & $\begin{array}{c}0.067^{* * *} \\
(0.014)\end{array}$ & $\begin{array}{c}0.080^{* * *} \\
(0.022)\end{array}$ \\
\hline Relative income & $\begin{array}{c}0.069^{\star * \star *} \\
(0.015)\end{array}$ & $\begin{array}{c}0.062^{\star \star \star \star} \\
(0.013)\end{array}$ & $\begin{array}{l}0.046^{\star *} \\
(0.019)\end{array}$ & $\begin{array}{c}0.075^{\star * \star} \\
(0.015)\end{array}$ & $\begin{array}{c}0.068^{* * *} \\
(0.019)\end{array}$ \\
\hline Observations & 6,699 & 6,983 & 3,507 & 6,599 & 3,576 \\
\hline$R$-squared & .150 & .156 & .185 & .152 & .186 \\
\hline \multicolumn{6}{|c|}{ B. Mental well-being } \\
\hline Dependent variable & \multicolumn{2}{|c|}{ Gender } & & Age & \\
\hline \multirow[t]{2}{*}{$\begin{array}{l}\text { Percentile of the } \\
\text { CES-D score }\end{array}$} & Male & Female & $\begin{array}{l}\text { Young } \\
(16-39)\end{array}$ & $\begin{array}{l}\text { Middle } \\
(40-59)\end{array}$ & $\begin{array}{c}\text { Old } \\
\text { (60 and } \\
\text { older) }\end{array}$ \\
\hline & (1) & $(2)$ & (3) & (4) & (5) \\
\hline $\begin{array}{l}\text { Dependent variable } \\
\text { mean }\end{array}$ & 0.449 & 0.510 & 0.469 & 0.483 & 0.485 \\
\hline Visibility & $\begin{array}{c}-0.506^{*} \\
(0.263)\end{array}$ & $\begin{array}{c}-0.605^{\star *} \\
(0.289)\end{array}$ & $\begin{array}{l}-0.264 \\
(0.426)\end{array}$ & $\begin{array}{c}-0.577^{\star *} \\
(0.262)\end{array}$ & $\begin{array}{c}-0.812^{* *} \\
(0.335)\end{array}$ \\
\hline Sunshine & $\begin{array}{c}0.168 \\
(0.366)\end{array}$ & $\begin{array}{c}0.191 \\
(0.347)\end{array}$ & $\begin{array}{c}0.689 \\
(0.468)\end{array}$ & $\begin{array}{c}0.148 \\
(0.353)\end{array}$ & $\begin{array}{c}-0.912^{*} \\
(0.476)\end{array}$ \\
\hline Visibility $\times$ Sunshine & $\begin{array}{l}0.198^{*} \\
(0.104)\end{array}$ & $\begin{array}{l}0.238^{* *} \\
(0.114)\end{array}$ & $\begin{array}{c}0.096 \\
(0.168)\end{array}$ & $\begin{array}{l}0.230^{* *} \\
(0.103)\end{array}$ & $\begin{array}{l}0.326^{\star *} \\
(0.131)\end{array}$ \\
\hline Per capita income & $\begin{array}{l}-0.002 \\
(0.004)\end{array}$ & $\begin{array}{c}0.003 \\
(0.004)\end{array}$ & $\begin{array}{l}-0.000 \\
(0.006)\end{array}$ & $\begin{array}{l}-0.005 \\
(0.005)\end{array}$ & $\begin{array}{c}0.008 \\
(0.006)\end{array}$ \\
\hline Relative income & $\begin{array}{c}-0.019^{* * *} \\
(0.004)\end{array}$ & $\begin{array}{c}-0.018^{* * *} \\
(0.004)\end{array}$ & $\begin{array}{c}-0.015^{\star *} \\
(0.006)\end{array}$ & $\begin{array}{c}-0.021^{* * *} \\
(0.004)\end{array}$ & $\begin{array}{c}-0.015^{\star \star *} \\
(0.006)\end{array}$ \\
\hline Observations & 13,304 & 13,766 & 7,550 & 13,067 & 6,453 \\
\hline (Within) $R$-squared & .041 & .032 & .056 & .032 & .028 \\
\hline
\end{tabular}

Source: Panel A is from the China Family Panel Studies (ISSS 2012), and panel B is from China Family Panel Studies (ISSS 2010; 2012).

Note: $\quad$ CES-D = Center for Epidemiologic Studies Depression scale. Lower percentile of the CES-D score indicates better mental well-being. In panel A, other covariates and fixed effects are the same as those in column (4) of Table 4.2. In panel B, other covariates and fixed effects are the same as those in column (4) of Table 4.3. Robust standard errors, clustered at the county level, are presented in parentheses. $* 10 \%$ significance level. $* * 5 \%$ significance level. $* * * 1 \%$ significance level.

If air quality is a normal good, we would expect that sensitivity to air pollution would increase with income. To test this hypothesis, we divide the respondents into four groups by quintiles of household per capita income. Results in Table 4.8 confirm that the poor are less responsive to air quality than are the rich. 
Table 4.8 Heterogeneity test of air quality effect, by income levels

\begin{tabular}{|c|c|c|c|c|}
\hline \multicolumn{5}{|c|}{ A. Hedonic happiness } \\
\hline Dependent variable & $0-25 \%$ & $25-50 \%$ & $50-75 \%$ & $75-100 \%$ \\
\hline Hedonic happiness & (1) & $(2)$ & (3) & $(4)$ \\
\hline $\begin{array}{l}\text { Dependent variable } \\
\text { mean }\end{array}$ & 2.697 & 2.795 & 2.892 & 2.990 \\
\hline Visibility & $\begin{array}{c}2.276 \\
(1.522)\end{array}$ & $\begin{array}{l}1.724^{* *} \\
(0.866)\end{array}$ & $\begin{array}{l}1.178 \\
(0.789)\end{array}$ & $\begin{array}{l}2.963^{* * *} \\
(0.847)\end{array}$ \\
\hline Sunshine & $\begin{array}{c}1.898 \\
(2.496)\end{array}$ & $\begin{array}{c}1.213 \\
(1.487)\end{array}$ & $\begin{array}{l}-0.430 \\
(1.585)\end{array}$ & $\begin{array}{c}1.852 \\
(1.375)\end{array}$ \\
\hline Visibility $\times$ Sunshine & $\begin{array}{l}-0.883 \\
(0.608)\end{array}$ & $\begin{array}{l}-0.626^{*} \\
(0.351)\end{array}$ & $\begin{array}{l}-0.432 \\
(0.309)\end{array}$ & $\begin{array}{c}-1.147^{\star \star *} \\
(0.335)\end{array}$ \\
\hline Per capita income & $\begin{array}{c}0.012 \\
(0.018)\end{array}$ & $\begin{array}{c}0.077 \\
(0.105)\end{array}$ & $\begin{array}{c}0.060 \\
(0.113)\end{array}$ & $\begin{array}{l}0.094^{* *} \\
(0.045)\end{array}$ \\
\hline Relative income & $\begin{array}{l}0.096^{* * *} \\
(0.020)\end{array}$ & $\begin{array}{l}0.065^{\star * *} \\
(0.019)\end{array}$ & $\begin{array}{c}0.033 \\
(0.020)\end{array}$ & $\begin{array}{l}0.050^{* * *} \\
(0.019)\end{array}$ \\
\hline Observations & 3,107 & 3,259 & 3,428 & 3,888 \\
\hline$R$-squared & .182 & .202 & .160 & .144 \\
\hline \multicolumn{5}{|c|}{ B. Mental well-being } \\
\hline Dependent variable & $0-25 \%$ & $25-50 \%$ & $50-75 \%$ & $75-100 \%$ \\
\hline $\begin{array}{l}\text { Percentile of the } \\
\text { CES-D score }\end{array}$ & (1) & (2) & (3) & (4) \\
\hline $\begin{array}{l}\text { Dependent variable } \\
\text { mean }\end{array}$ & 0.547 & 0.502 & 0.468 & 0.421 \\
\hline Visibility & $\begin{array}{l}-0.681 \\
(0.525)\end{array}$ & $\begin{array}{c}-0.646^{* *} \\
(0.320)\end{array}$ & $\begin{array}{l}-0.646 \\
(0.410)\end{array}$ & $\begin{array}{c}-1.200^{* * *} \\
(0.416)\end{array}$ \\
\hline Sunshine & $\begin{array}{l}-0.436 \\
(0.724)\end{array}$ & $\begin{array}{c}0.173 \\
(0.594)\end{array}$ & $\begin{array}{c}0.793 \\
(0.527)\end{array}$ & $\begin{array}{l}-0.344 \\
(0.490)\end{array}$ \\
\hline Visibility $\times$ Sunshine & $\begin{array}{c}0.268 \\
(0.207)\end{array}$ & $\begin{array}{l}0.255^{\star *} \\
(0.128)\end{array}$ & $\begin{array}{c}0.246 \\
(0.164)\end{array}$ & $\begin{array}{l}0.476^{* * *} \\
(0.164)\end{array}$ \\
\hline Per capita income & $\begin{array}{c}0.012 \\
(0.009)\end{array}$ & $\begin{array}{l}-0.021 \\
(0.040)\end{array}$ & $\begin{array}{l}-0.034 \\
(0.052)\end{array}$ & $\begin{array}{c}-0.030^{* *} \\
(0.014)\end{array}$ \\
\hline Relative income & $\begin{array}{c}-0.029^{* * *} \\
(0.008)\end{array}$ & $\begin{array}{c}-0.021^{* *} \\
(0.009)\end{array}$ & $\begin{array}{c}-0.024^{* * *} \\
(0.009)\end{array}$ & $\begin{array}{l}-0.001 \\
(0.007)\end{array}$ \\
\hline $\begin{array}{l}\text { Observations } \\
\text { (Within) } R \text {-squared }\end{array}$ & $\begin{array}{c}5,969 \\
.058\end{array}$ & $\begin{array}{l}6,490 \\
.063\end{array}$ & $\begin{array}{c}6,727 \\
.081\end{array}$ & $\begin{array}{c}7,884 \\
.070\end{array}$ \\
\hline
\end{tabular}

Source: Panel A is from the China Family Panel Studies (ISSS 2012), and panel B is from China Family Panel Studies (ISSS 2010; 2012).

Note: $\quad$ CES-D = Center for Epidemiologic Studies Depression scale. Lower percentile of the CES-D score indicates better mental well-being. In panel A, other covariates and fixed effects are the same as those in column (4) of Table 4.2. In panel B, other covariates and fixed effects are the same as those in column (4) of Table 4.3. Robust standard errors, clustered at the county level, are presented in parentheses. $* 10 \%$ significance level. $* * 5 \%$ significance level. $* * * 1 \%$ significance level.

Attitude toward pollution may also affect reaction to air quality. CFPS 2012 includes a host of questions to elicit people's attitudes toward major social issues in China, such as inequality, environmental quality, social security, education, and corruption. ${ }^{10}$ The first two columns of Table 4.9 divide the sample by attitude toward environmental quality, and results suggest that air quality imposes larger and more significantly positive effects on people who are more critical about environmental issues.

10 The question about the environmental issue is framed as, "How severe do you think is the environmental problem in China?" The answer is rated by a number ranging from 0 (not severe at all) to 10 (very severe). To mitigate the concern that some respondents may overstate or understate their general attitudes toward social issues, we calculate a normalized attitude score toward pollution by dividing the pollution assessment score by the average ratings of all eight questions. We divide our sample into two groups by the median of the normalized score. 
Table 4.9 Heterogeneity test of air quality effect, by pollution attitude and workplace

\begin{tabular}{|c|c|c|c|c|}
\hline \multicolumn{5}{|c|}{ A. Hedonic happiness } \\
\hline \multirow{3}{*}{$\begin{array}{l}\text { Dependent variable } \\
\text { Hedonic happiness }\end{array}$} & \multicolumn{2}{|c|}{ Pollution attitude } & \multicolumn{2}{|c|}{ Workplace } \\
\hline & Careless & Critical & Indoors & Outdoors \\
\hline & (1) & (2) & (3) & (4) \\
\hline Dependent variable mean & 2.838 & 2.888 & 2.962 & 2.818 \\
\hline Visibility & $\begin{array}{l}1.541^{*} \\
(0.832)\end{array}$ & $\begin{array}{c}2.127^{* * *} \\
(0.642)\end{array}$ & $\begin{array}{c}0.028 \\
(0.813)\end{array}$ & $\begin{array}{l}2.187^{* *} \\
(1.034)\end{array}$ \\
\hline Sunshine & 1.655 & -0.253 & 0.453 & 0.399 \\
\hline \multirow{2}{*}{ Visibility $\times$ Sunshine } & $\begin{array}{l}(1.435) \\
-0.595^{*}\end{array}$ & $\begin{array}{c}(1.111) \\
-0.800^{* * *}\end{array}$ & $\begin{array}{c}(1.505) \\
0.025\end{array}$ & $\begin{array}{l}(1.529) \\
-0.854^{* *}\end{array}$ \\
\hline & $(0.335)$ & $(0.256)$ & $(0.334)$ & $(0.412)$ \\
\hline \multirow[t]{2}{*}{ Per capita income } & $0.050^{* * *}$ & $0.057^{* * *}$ & $0.059^{* * *}$ & $0.041^{* * *}$ \\
\hline & $(0.014)$ & $(0.012)$ & $(0.019)$ & $(0.011)$ \\
\hline \multirow[t]{2}{*}{ Relative income } & $0.071^{* * *}$ & $0.054^{* * *}$ & $0.043^{* *}$ & $0.069^{* * *}$ \\
\hline & $(0.013)$ & $(0.014)$ & $(0.019)$ & $(0.013)$ \\
\hline Observations & 6,260 & 6,878 & 3,718 & 6,242 \\
\hline$R$-squared & .160 & .153 & .156 & .164 \\
\hline \multicolumn{5}{|c|}{ B. Mental well-being } \\
\hline \multirow{3}{*}{$\begin{array}{l}\text { Dependent variable } \\
\text { Percentile of the } \\
\text { CES-D score }\end{array}$} & \multicolumn{2}{|c|}{ Pollution attitude } & \multicolumn{2}{|c|}{ Workplace } \\
\hline & Careless & Critical & Indoors & Outdoors \\
\hline & (1) & $(2)$ & (3) & (4) \\
\hline Dependent variable mean & 0.484 & 0.470 & 0.445 & 0.485 \\
\hline \multirow[t]{2}{*}{ Visibility } & -0.377 & $-0.687^{* *}$ & $-0.648^{* *}$ & $-0.467^{* \star}$ \\
\hline & $(0.263)$ & $(0.301)$ & $(0.307)$ & $(0.203)$ \\
\hline \multirow[t]{2}{*}{ Sunshine } & 0.340 & 0.105 & 0.453 & 0.373 \\
\hline & $(0.351)$ & $(0.367)$ & $(0.502)$ & $(0.338)$ \\
\hline \multirow[t]{2}{*}{ Visibility $\times$ Sunshine } & 0.146 & $0.273^{* *}$ & $0.245^{* *}$ & $0.189^{* *}$ \\
\hline & $(0.103)$ & $(0.119)$ & $(0.122)$ & $(0.080)$ \\
\hline \multirow[t]{2}{*}{ Per capita income } & -0.001 & -0.000 & -0.011 & -0.005 \\
\hline & $(0.005)$ & $(0.005)$ & $(0.009)$ & $(0.006)$ \\
\hline \multirow[t]{2}{*}{ Relative income } & $-0.019^{* * *}$ & $-0.015^{\star \star \star}$ & $-0.023^{* *}$ & $-0.023^{\star \star *}$ \\
\hline & $(0.004)$ & $(0.004)$ & $(0.009)$ & $(0.005)$ \\
\hline Observations & 12,354 & 13,626 & 6,175 & 10,220 \\
\hline (Within) $R$-squared & .030 & .035 & .088 & .040 \\
\hline
\end{tabular}

Source: Panel A is from the China Family Panel Studies (ISSS 2012), and panel B is from China Family Panel Studies (ISSS 2010; 2012).

Note: $\quad$ CES-D = Center for Epidemiologic Studies Depression scale. Lower percentile of the CES-D score indicates better mental well-being. In panel A, other covariates and fixed effects are the same as those in column (4) of Table 4.2. In panel B, other covariates and fixed effects are the same as those in column (4) of Table 4.3. Robust standard errors, clustered at the county level, are presented in parentheses. $* 10 \%$ significance level. $* * 5 \%$ significance level. $* * * 1 \%$ significance level.

Workplace may play an important role in affecting people's response to air quality. An increase in particulate matter outdoors even leads to a significant reduction of indoor worker productivity (Chang et al. 2014; Li, Liu, and Salvo 2015). We test whether air quality imposes large impacts on the SWB of people working outdoors and indoors. Results presented in the last two columns of Table 4.9 show that bad air quality reduces hedonic happiness only among people who work outdoors, but it affects the mental well-being of all people no matter whether they work indoors or outdoors. 
Do people living in more polluted areas respond to air pollution differently from those in less polluted areas? On the one hand, both the dose-response relationship and increasing marginal disutility from air pollution may mean that heavier air pollution generates larger negative impacts on SWB. On the other hand, people in more polluted areas could be less sensitive to air pollution either because they are habituated to the poor air quality or because people who are less concerned about air pollution sort into more polluted areas ex ante. We divide the whole sample by the median level of yearly average visibility. Results in columns (1) through (2) of Table 4.10, similar to Levinson (2012), show significant effects of air pollution only in less polluted areas, indicating that habituation and potential self-selection may dominate the dose-response relationship between air pollution and SWB.

Table 4.10 Heterogeneity test of air quality effect, by pollution level and health

\begin{tabular}{|c|c|c|c|c|}
\hline \multicolumn{5}{|c|}{ A. Hedonic happiness } \\
\hline \multirow{3}{*}{$\begin{array}{l}\text { Dependent variable } \\
\text { Hedonic happiness }\end{array}$} & \multicolumn{2}{|c|}{ Local yearly pollution level } & \multicolumn{2}{|c|}{ Chronic disease } \\
\hline & Polluted & Less polluted & Yes & No \\
\hline & $(1)$ & $(2)$ & (3) & (4) \\
\hline $\begin{array}{l}\text { Dependent variable } \\
\text { mean }\end{array}$ & 2.882 & 2.833 & 2.741 & 2.872 \\
\hline Visibility & $\begin{array}{c}1.306 \\
(1.657)\end{array}$ & $\begin{array}{l}2.193^{* * *} \\
(0.660)\end{array}$ & $\begin{array}{l}2.922^{* *} \\
(1.271)\end{array}$ & $\begin{array}{l}1.683^{* *} \\
(0.751)\end{array}$ \\
\hline Sunshine & $\begin{array}{c}0.488 \\
(1.667)\end{array}$ & $\begin{array}{c}0.472 \\
(1.264)\end{array}$ & $\begin{array}{l}4.289^{*} \\
(2.404)\end{array}$ & $\begin{array}{c}0.059 \\
(1.161)\end{array}$ \\
\hline Visibility $\times$ Sunshine & $\begin{array}{l}-0.485 \\
(0.655)\end{array}$ & $\begin{array}{c}-0.845^{* * *} \\
(0.265)\end{array}$ & $\begin{array}{c}-1.111^{* *} \\
(0.508)\end{array}$ & $\begin{array}{c}-0.641^{* *} \\
(0.300)\end{array}$ \\
\hline Per capita income & $\begin{array}{c}0.068^{\star * \star} \\
(0.016)\end{array}$ & $\begin{array}{c}0.045^{\star * *} \\
(0.012)\end{array}$ & $\begin{array}{l}0.055^{\star *} \\
(0.023)\end{array}$ & $\begin{array}{c}0.056^{* * *} \\
(0.011)\end{array}$ \\
\hline Relative income & $\begin{array}{c}0.074^{* * *} \\
(0.015)\end{array}$ & $\begin{array}{l}0.055^{* * *} \\
(0.012)\end{array}$ & $\begin{array}{c}0.085^{\star * *} \\
(0.026)\end{array}$ & $\begin{array}{c}0.063^{* * *} \\
(0.010)\end{array}$ \\
\hline $\begin{array}{l}\text { Observations } \\
R \text {-squared }\end{array}$ & $\begin{array}{c}6,598 \\
.118 \\
\end{array}$ & $\begin{array}{c}6,658 \\
.184 \\
\end{array}$ & $\begin{array}{c}2,005 \\
.181 \\
\end{array}$ & $\begin{array}{c}11,677 \\
.154 \\
\end{array}$ \\
\hline \multicolumn{5}{|c|}{ B. Mental well-being } \\
\hline Dependent variable & \multicolumn{2}{|c|}{ Local yearly pollution level } & \multicolumn{2}{|c|}{ Chronic disease } \\
\hline $\begin{array}{l}\text { Percentile of the } \\
\text { CES-D score }\end{array}$ & $\begin{array}{c}\text { Polluted } \\
\text { (1) }\end{array}$ & $\begin{array}{c}\text { Less polluted } \\
(2)\end{array}$ & $\begin{array}{l}\text { Yes } \\
(3)\end{array}$ & $\begin{array}{l}\text { No } \\
(4)\end{array}$ \\
\hline $\begin{array}{l}\text { Dependent variable } \\
\text { mean }\end{array}$ & 0.456 & 0.500 & 0.572 & 0.464 \\
\hline Visibility & $\begin{array}{l}-0.356 \\
(0.269)\end{array}$ & $\begin{array}{c}-0.944^{* *} \\
(0.435)\end{array}$ & $\begin{array}{c}-1.050^{* *} \\
(0.406)\end{array}$ & $\begin{array}{l}-0.382 \\
(0.252)\end{array}$ \\
\hline Sunshine & $\begin{array}{c}0.356 \\
(0.408)\end{array}$ & $\begin{array}{c}0.040 \\
(0.525)\end{array}$ & $\begin{array}{c}-1.382^{* *} \\
(0.603)\end{array}$ & $\begin{array}{c}0.409 \\
(0.323)\end{array}$ \\
\hline Visibility $\times$ Sunshine & $\begin{array}{c}0.137 \\
(0.106)\end{array}$ & $\begin{array}{l}0.375^{* *} \\
(0.172)\end{array}$ & $\begin{array}{l}0.402^{* *} \\
(0.160)\end{array}$ & $\begin{array}{c}0.148 \\
(0.099)\end{array}$ \\
\hline Per capita income & $\begin{array}{c}0.002 \\
(0.005)\end{array}$ & $\begin{array}{l}-0.000 \\
(0.005)\end{array}$ & $\begin{array}{l}-0.007 \\
(0.012)\end{array}$ & $\begin{array}{c}0.001 \\
(0.004)\end{array}$ \\
\hline Relative income & $\begin{array}{c}-0.013^{* *} \\
(0.005)\end{array}$ & $\begin{array}{c}-0.020^{* * *} \\
(0.004)\end{array}$ & $\begin{array}{l}-0.018 \\
(0.011)\end{array}$ & $\begin{array}{c}-0.015^{\star * *} \\
(0.004)\end{array}$ \\
\hline $\begin{array}{l}\text { Observations } \\
\text { (Within) } R \text {-squared }\end{array}$ & $\begin{array}{c}13,132 \\
.026\end{array}$ & $\begin{array}{c}13,228 \\
.053\end{array}$ & $\begin{array}{l}4,031 \\
.096\end{array}$ & $\begin{array}{c}23,039 \\
.024\end{array}$ \\
\hline
\end{tabular}

Source: Panel A is from the China Family Panel Studies (ISSS 2012), and panel B is from China Family Panel Studies (ISSS 2010; 2012).

Note: $\quad$ CES-D = Center for Epidemiologic Studies Depression scale. Lower percentile of the CES-D score indicates better mental well-being. In panel A, other covariates and fixed effects are the same as those in column (4) of Table 4.2. In panel B, other covariates and fixed effects are the same as those in column (4) of Table 4.3. Robust standard errors, clustered at the county level, are presented in parentheses. $* 10 \%$ significance level. $* * 5 \%$ significance level. $* * * 1 \%$ significance level. 
We expect people in poor health to be more emotionally vulnerable to air pollution. Columns (3) through (4) of Table 4.10 divide the sample by chronic disease status. Those who suffer from chronic diseases, such as asthma and heart attacks, demonstrate more salient negative impacts of air pollution.

Moreover, less educated people may be less concerned about air quality as low education may restrict their ability to acquire information about air quality through, for example, smart phones or the Internet (Levinson 2012; Greenstone and Hanna 2014). We test this hypothesis taking advantage of two sources of air pollution data. Air visibility is presumably easier to perceive than API as the latter requires additional information and certain knowledge. Dividing the whole sample by education, Table 4.11 indicates that those who receive more education react more to both visibility and API than do the less educated.

Table 4.11 Heterogeneity test of air quality effect, by education years

\begin{tabular}{|c|c|c|c|c|}
\hline \multicolumn{5}{|c|}{ A. Hedonic happiness } \\
\hline Dependent variable & \multicolumn{2}{|c|}{ Visibility } & \multicolumn{2}{|c|}{ API } \\
\hline Hedonic happiness & $\begin{array}{l}\text { Less educated } \\
\text { (education } \leq 6 \text { ) }\end{array}$ & $\begin{array}{l}\text { More educated } \\
\text { (education } \geq 9)\end{array}$ & $\begin{array}{l}\text { Less educated } \\
\text { (education } \leq 6 \text { ) }\end{array}$ & $\begin{array}{l}\text { More educated } \\
\text { (education } \geq 9 \text { ) }\end{array}$ \\
\hline & (1) & $(2)$ & (3) & (4) \\
\hline Dependent variable mean & 2.711 & 2.976 & 2.713 & 2.976 \\
\hline Air quality & $\begin{array}{c}1.546 \\
(0.941)\end{array}$ & $\begin{array}{l}2.175^{\star * *} \\
(0.677)\end{array}$ & $\begin{array}{c}0.326 \\
(1.278)\end{array}$ & $\begin{array}{c}-2.320^{* * *} \\
(0.706)\end{array}$ \\
\hline Sunshine & $\begin{array}{c}0.951 \\
(1.908)\end{array}$ & $\begin{array}{l}-0.111 \\
(1.064)\end{array}$ & $\begin{array}{c}0.347 \\
(2.192)\end{array}$ & $\begin{array}{l}-5.494^{* * *} \\
(1.323)\end{array}$ \\
\hline Air quality $\times$ Sunshine & $\begin{array}{l}-0.562 \\
(0.374)\end{array}$ & $\begin{array}{c}-0.837^{* * *} \\
(0.271)\end{array}$ & $\begin{array}{l}-0.139 \\
(0.507)\end{array}$ & $\begin{array}{l}0.891^{* * *} \\
(0.287)\end{array}$ \\
\hline Per capita income & $\begin{array}{l}0.068^{* * *} \\
(0.013)\end{array}$ & $\begin{array}{l}0.049^{* * *} \\
(0.013)\end{array}$ & $\begin{array}{l}0.066^{* * *} \\
(0.013)\end{array}$ & $\begin{array}{l}0.048^{* * *} \\
(0.013)\end{array}$ \\
\hline Relative income & $\begin{array}{c}0.074^{* * *} \\
(0.013)\end{array}$ & $\begin{array}{l}0.051^{* * *} \\
(0.015)\end{array}$ & $\begin{array}{l}0.075^{* * *} \\
(0.013)\end{array}$ & $\begin{array}{l}0.049^{* * *} \\
(0.014)\end{array}$ \\
\hline Observations & 5,933 & 6,615 & 5,972 & 6,626 \\
\hline$R$-squared & .174 & .131 & .171 & .130 \\
\hline \multicolumn{5}{|c|}{ B. Mental well-being } \\
\hline Dependent variable & \multicolumn{2}{|c|}{ Visibility } & \multicolumn{2}{|c|}{ API } \\
\hline $\begin{array}{l}\text { Percentile of the } \\
\text { CES-D score }\end{array}$ & $\begin{array}{l}\text { Less educated } \\
\text { (education } \leq 6 \text { ) }\end{array}$ & $\begin{array}{l}\text { More educated } \\
\text { (education } \geq 9)\end{array}$ & $\begin{array}{l}\text { Less educated } \\
\text { (education } \leq 6 \text { ) }\end{array}$ & $\begin{array}{l}\text { More educated } \\
\text { (education } \geq 9 \text { ) }\end{array}$ \\
\hline & (1) & $(2)$ & (3) & (4) \\
\hline Dependent variable mean & 0.527 & 0.435 & 0.529 & 0.432 \\
\hline Air quality & $\begin{array}{l}-0.510^{*} \\
(0.268)\end{array}$ & $\begin{array}{c}-0.659^{* *} \\
(0.303)\end{array}$ & $\begin{array}{l}0.788^{*} \\
(0.466)\end{array}$ & $\begin{array}{l}1.198^{* *} \\
(0.537)\end{array}$ \\
\hline Sunshine & $\begin{array}{l}-0.101 \\
(0.342)\end{array}$ & $\begin{array}{c}0.129 \\
(0.421)\end{array}$ & $\begin{array}{l}1.587^{\star} \\
(0.844)\end{array}$ & $\begin{array}{c}2.634^{* * *} \\
(0.843)\end{array}$ \\
\hline Air quality $\times$ Sunshine & $\begin{array}{l}0.199^{\star} \\
(0.106)\end{array}$ & $\begin{array}{l}0.260^{* *} \\
(0.119)\end{array}$ & $\begin{array}{l}-0.306^{*} \\
(0.183)\end{array}$ & $\begin{array}{c}-0.472^{* *} \\
(0.212)\end{array}$ \\
\hline Per capita income & $\begin{array}{c}0.003 \\
(0.005)\end{array}$ & $\begin{array}{l}-0.001 \\
(0.005)\end{array}$ & $\begin{array}{c}0.007 \\
(0.006)\end{array}$ & $\begin{array}{l}-0.001 \\
(0.005)\end{array}$ \\
\hline Relative income & $\begin{array}{c}-0.018^{* * *} \\
(0.004)\end{array}$ & $\begin{array}{c}-0.015^{\star * *} \\
(0.004)\end{array}$ & $\begin{array}{c}-0.016^{* * *} \\
(0.005)\end{array}$ & $\begin{array}{c}-0.014^{\star * *} \\
(0.005)\end{array}$ \\
\hline Observations & 12,467 & 12,751 & 11,138 & 11,570 \\
\hline (Within) $R$-squared & .027 & .045 & .025 & .045 \\
\hline
\end{tabular}

Source: Panel A is from the China Family Panel Studies (ISSS 2012), and panel B is from China Family Panel Studies (ISSS 2010; 2012).

Note: $\quad$ API $=$ air pollution index; CES-D = Center for Epidemiologic Studies Depression scale. Lower percentile of the CES-D score indicates better mental well-being. In panel A, other covariates and fixed effects are the same as those in column (4) of Table 4.2. In panel B, other covariates and fixed effects are the same as those in column (4) of Table 4.3. Robust standard errors, clustered at the county level, are presented in parentheses. ${ }^{*} 10 \%$ significance level. $* * 5 \%$ significance level. ***1\% significance level. 
Finally, it is likely that households with children are more sensitive to air pollution. We separate the sample by whether a household has young children (that is, children younger than age 16) but find no distinguishable differences between the two. 


\section{IMPLICATIONS FOR THE EASTERLIN PARADOX}

Following the seminal work of Easterlin $(1974,1995)$, there has been a growing literature explaining the happiness puzzle, also known as the Easterlin paradox, that income growth does not necessarily improve (hedonic) happiness. ${ }^{11}$ Concern for relative income has been regarded as the most plausible explanation for the paradox (Luttmer 2005; Clark, Frijters, and Shields 2008; Chen 2015b). Other plausible driving forces include the framing of happiness questions (Graham, Chattopadhyay, and Picon 2010), income measurement errors (Graham, Chattopadhyay, and Picon 2010), and omitted factors (for example, social trust and freedom) (Helliwell 2012; Inglehart et al. 2008).

In this paper, we argue for the first time that air pollution is another plausible contributor to the paradox. We compute the economic significance of air pollution, relative income, and other correlates on the declining level of happiness and evaluate their importance in explaining the Easterlin paradox in China.

China provides a good setting to study the Easterlin paradox. In spite of unprecedented income growth, China's average happiness measures did not improve from 1990 to 2010 (Easterlin et al. 2012). Life satisfaction (scaled from 1, dissatisfied, to 10, satisfied) and happiness (scaled from 1, not at all happy, to 4, very happy) of the World Value Survey, happiness (scaled from 1, very unhappy, to 5, very happy) of the China Central Television (CCTV) Postcard Survey, and urban life satisfaction (scaled from 1 , very dissatisfied, to 5, very satisfied) of the Horizon Research all reveal an obvious pattern of stagnant or even decreasing happiness in China during past decades. For example, the Horizon Research indicates that life satisfaction fell from 3.69 in 1997 to 3.57 in 2012 (Table A.2). The CCTV survey finds that the proportion of people feeling happy or very happy declined from 54.1 percent to 48.0 percent between 2007 and 2012, while the share of people reporting they are unhappy or very unhappy rose from 7.6 percent to 11.6 percent (Figure A.1).

Easterlin et al. (2012) attribute the Chinese happiness puzzle to a high unemployment rate due to state-owned enterprise (SOE) reforms, the dissolution of the social safety net, and rising inequality throughout the 1990s and the early 2000s. These factors may explain the temporal pattern from 1990 through 2007, but they cannot account for the decline in happiness during 2007 through 2012, as all these indicators were improved in that time. The SOE reforms were finished by the late 1990s. Since 2004, the labor market has become tighter, resulting in lower unemployment rates and more rapid increases in real wages (Zhang, Yang, and Wang 2011; Zhang et al. 2014). Along with lower unemployment rates and rising wages, the Gini coefficient for China as a whole has declined since 2008 (Xie et al. 2013). Moreover, more social safety nets have been put in place in the past decade. For example, the new rural cooperative medical insurance program has been gradually scaled up in rural areas since 2003 (Li, Xia, and $\mathrm{Yu}$ 2014), and the new rural pension program has been rolled out since 2009 (Chen 2015a). The evidence suggests additional factors are at play.

We echo Easterlin (1974) by finding a noticeable positive association between absolute income and happiness across individuals within a county at a given point of time (Table 4.2), but the association disappears over time (Table 4.3). However, relative income affects hedonic well-being both at a point of time and over time, confirming relative income as a plausible explanation to the Easterlin paradox.

More interesting, our results lend support to worsening air quality as an additional driving force for the observed decline in happiness. According to the World Bank, 16 of the world's top 20 most polluted cities are in China. ${ }^{12}$ The report published by China's Ministry of Environmental Protection in June 2013 shows that about 60 percent of 325 prefecture-level cities failed to meet the Ambient Air

\footnotetext{
${ }^{11}$ Several empirical studies (Hagerty and Veenhoven 2003; Stevenson and Wolfers 2008) based on cross-country data dismiss the paradox. Drawing an updated database of 37 countries, Easterlin et al. (2010) reconfirm the paradox. Despite the controversial cross-country evidence, the paradox has been observed in a number of major economies (Tella and MacCulloch 2006; Easterlin et al. 2012).

${ }^{12}$ See www.cbsnews.com/news/the-most-polluted-places-on-earth/, citing “The Little Green Data Book” (World Bank, May 2007, ISBN 0-8213-6967-9).
} 
Quality Standards (GB3095-2012) in 2012. ${ }^{13}$ As revealed in Figure A.2, air visibility declined sharply from 1997 to 2012 in China.

Based on a back-of-the-envelope calculation using the results in Table 4.2, a one $S D$ increase in visibility lifts happiness by 0.043 (equivalent to $0.043 \mathrm{SDs}$ ). The impact is rather sizable considering that a one $S D$ increase in income status, one of the most important predictors of happiness, raises happiness by 0.064 (equivalent to $0.064 S D$ s). In the Horizon Research, self-reported happiness declined by $0.174 S D$ s (measured by the $S D$ in 2012) from 1997 to 2012. Seen from Figure A.2, the 0.95-mile or 0.268-standarddeviation (measured by the $S D$ in 2012) decline in visibility during the same period accounts for 6.6 percent of the actual decrease in happiness.

Finally, we assess the money metric value of air quality. By totally differentiating equation 1 and setting $d H=0$, we calculate the average marginal rate of substitution between air quality and absolute income $\partial Y /\left.\partial P\right|_{d H=0}=-Y\left(\hat{\alpha}_{1}+\hat{\alpha}_{3} \ln\right.$ sunshine $) /\left(P \hat{\beta}_{1}\right)$, also known as WTP. Plugging in 1.879 for $\hat{\alpha}_{1}$, -0.714 for $\hat{\alpha}_{3}, 0.055$ for $\hat{\beta}_{1}$ in Table 4.2 , and 13,264 for the mean income, 8.783 for the mean visibility, and 12.195 for the mean hours of sunshine, WTP amounts to CNY 2,562, revealing that a $0.1 S D$ improvement in air quality raises an average person's happiness by an amount worth CNY 1,041 $(2,562 \times$ $4.064 \times 0.1$ ) per year. ${ }^{14}$ This means people are willing to pay CNY 2.9 per day per person (or $\$ 170$ per year per person) for a $0.1 S D$ improvement in air quality. Our estimated WTP in a developing context is much smaller than those gauged by the US studies if measured in dollar terms. For example, Levinson (2012) estimates that an average person is willing to pay $\$ 1.80$ (or CNY 11.2) per day for a $0.1 S D$ improvement in air quality compared to CNY 2.9 in China. However, if measured as the share of WTP in gross domestic product (GDP) per capita, Chinese are even willing to pay a larger share of their income, 2.7 percent $(\mathrm{CNY} 2.9 \times 365 / \mathrm{CNY} 39,544)$, to reduce air pollution than their U.S. counterparts $(1.3$ percent $=\$ 1.8 \times 365 / \$ 51,457) .{ }^{15}$

${ }^{13}$ See www.zhb.gov.cn/gkml/hbb/qt/201306/t20130604_253201.htm.

${ }^{14}$ One standard deviation of visibility is 4.064 .

15 According to the World Development Indicators released by the World Bank, in 2012 gross domestic product per capita in China and the United States are CNY 39,544 and \$51,457, respectively. 


\section{CONCLUSION}

This paper estimates the impact of air quality on long-term life satisfaction, short-term hedonic happiness, and mental well-being by matching self-reported SWB measures in CFPS, a nationally representative survey, with air quality data from both internal and external sources according to the exact date of the interview. Our results show muted effects of daily air quality on overall life satisfaction, but bad air quality lowers hedonic happiness and raises the rate of depressive symptoms. In particular, people who are more concerned with environmental problems, work outdoors, earn higher incomes, reside in less polluted areas, or are in poor health are more sensitive to air quality.

Our paper shows worsening air quality may contribute to the Easterlin paradox in China. The impact of air quality on happiness is just slightly less than that of relative income, the currently accepted main contributor to the paradox. The findings suggest that the GDP-obsessed development strategy in China has not brought about more happiness. The government should go beyond GDP and include environmental indicators, such as air quality indexes, in cadres' evaluation packages.

Furthermore, evaluating a wider spectrum of the impact of air pollution is valuable to public policies. Our results indicate that the current emphasis on health-related costs understates the potential costs of pollution and therefore the benefits of reducing pollution. More scientific evidence is expected in the aspects of cognition, labor productivity, and SWB. 


\section{APPENDIX A: SUPPLEMENTARY FIGURES AND TABLES}

Figure A.1 Happiness trend according to the China Central Television (CCTV) Postcard Survey, 2007-2012

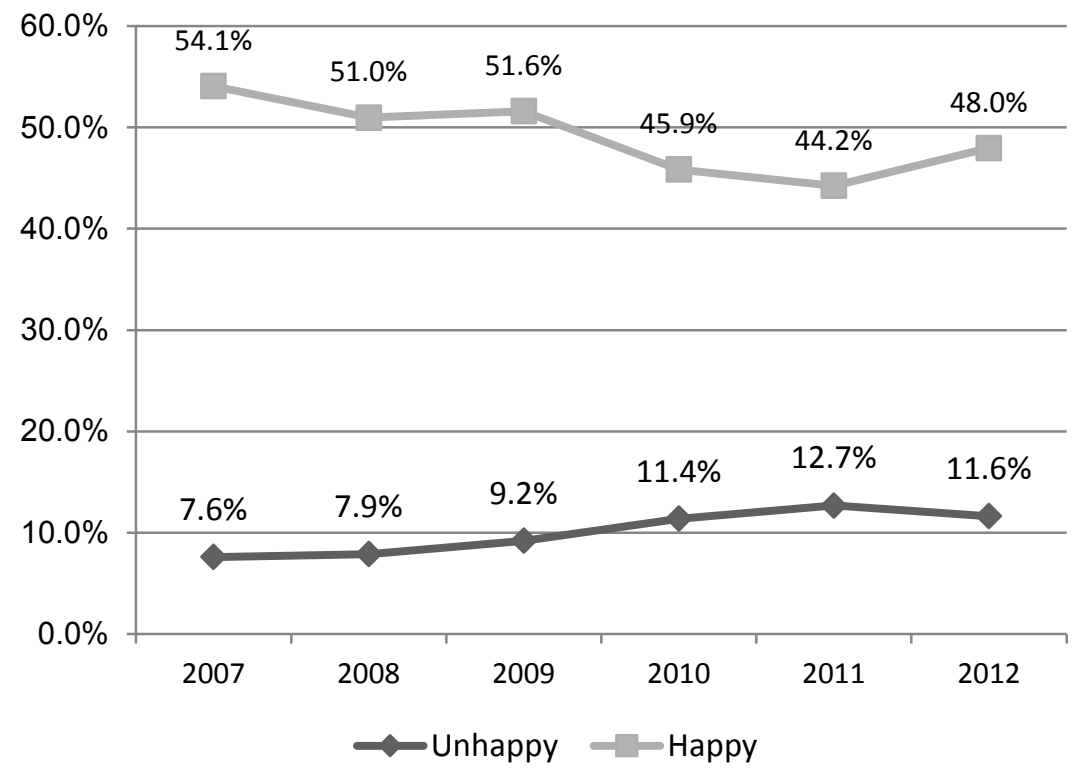

Source: CCTV Postcard Survey $(2007-2012)$.

Figure A.2 Visibility trend in China, 1997-2012

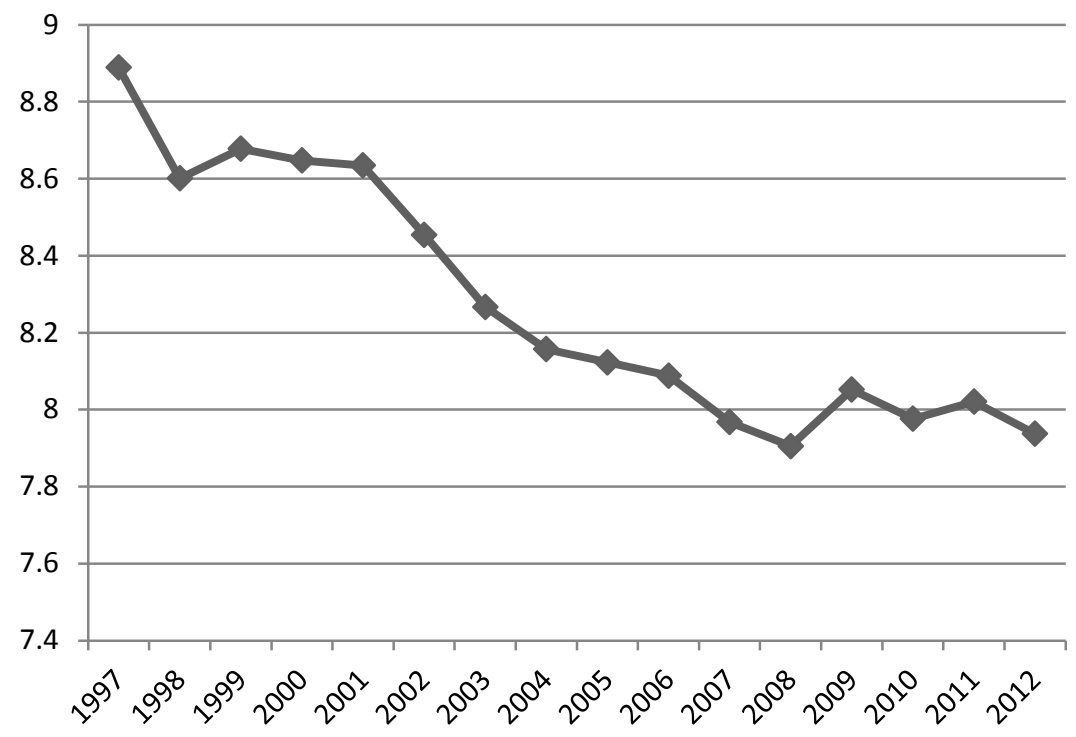

Source: National Oceanic and Atmospheric Administration (1997-2012).

Note: The yearly average visibility is calculated by the weighted average values of all the monitor stations in China where the weights are equal to the population at the county level. 
Figure A.3 Relation between visibility and $\mathrm{PM10}_{2} \mathrm{SO}_{2}$, and $\mathrm{NO}_{2}$

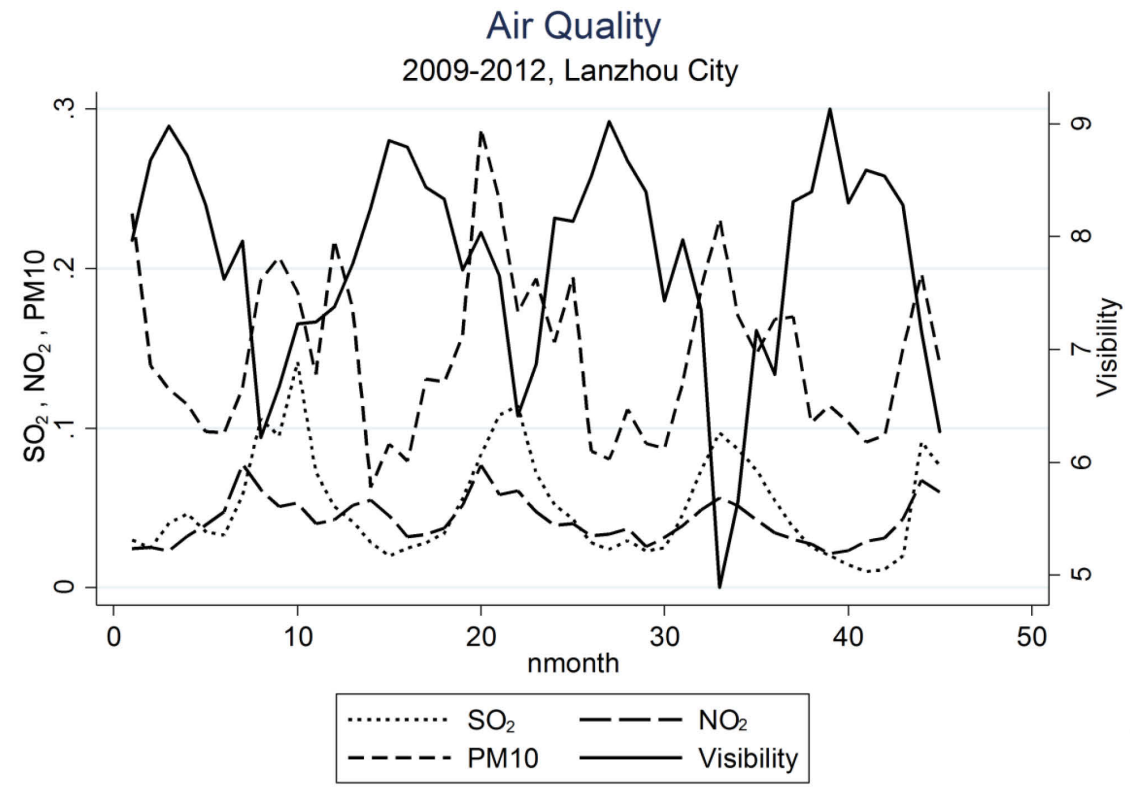

Source: Chen et al. (2015).

Note: $\quad$ Jan = January; Mar $=$ March; $\mathrm{NO}_{2}=$ nitrogen dioxide; Nov $=$ November; PM10 $=$ fine particulate matter smaller than 10 micrometers; Sep $=$ September; $\mathrm{SO}_{2}=$ sulfur dioxide.

Figure A.4 Interview date distribution, 2010 and 2012

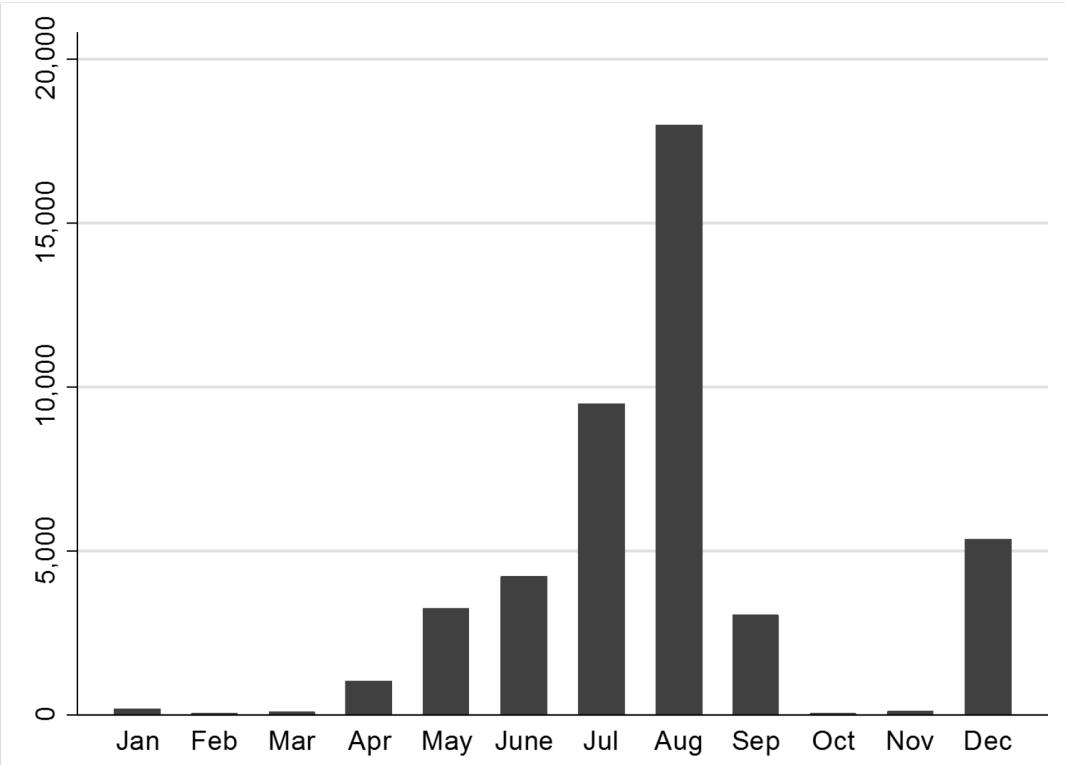

Source: China Family Panel Studies (ISSS 2010; 2012).

Note: $\quad$ Apr $=$ April; Aug $=$ August $;$ Dec $=$ December; Feb $=$ February; Jan $=$ January; Jul = July; Mar $=$ March; Nov $=$ November; Oct $=$ October; Sep $=$ September. 
Figure A.5 The Baidu Index of "wumai (haze)" and "kouzhao (mask)", 2012-2013
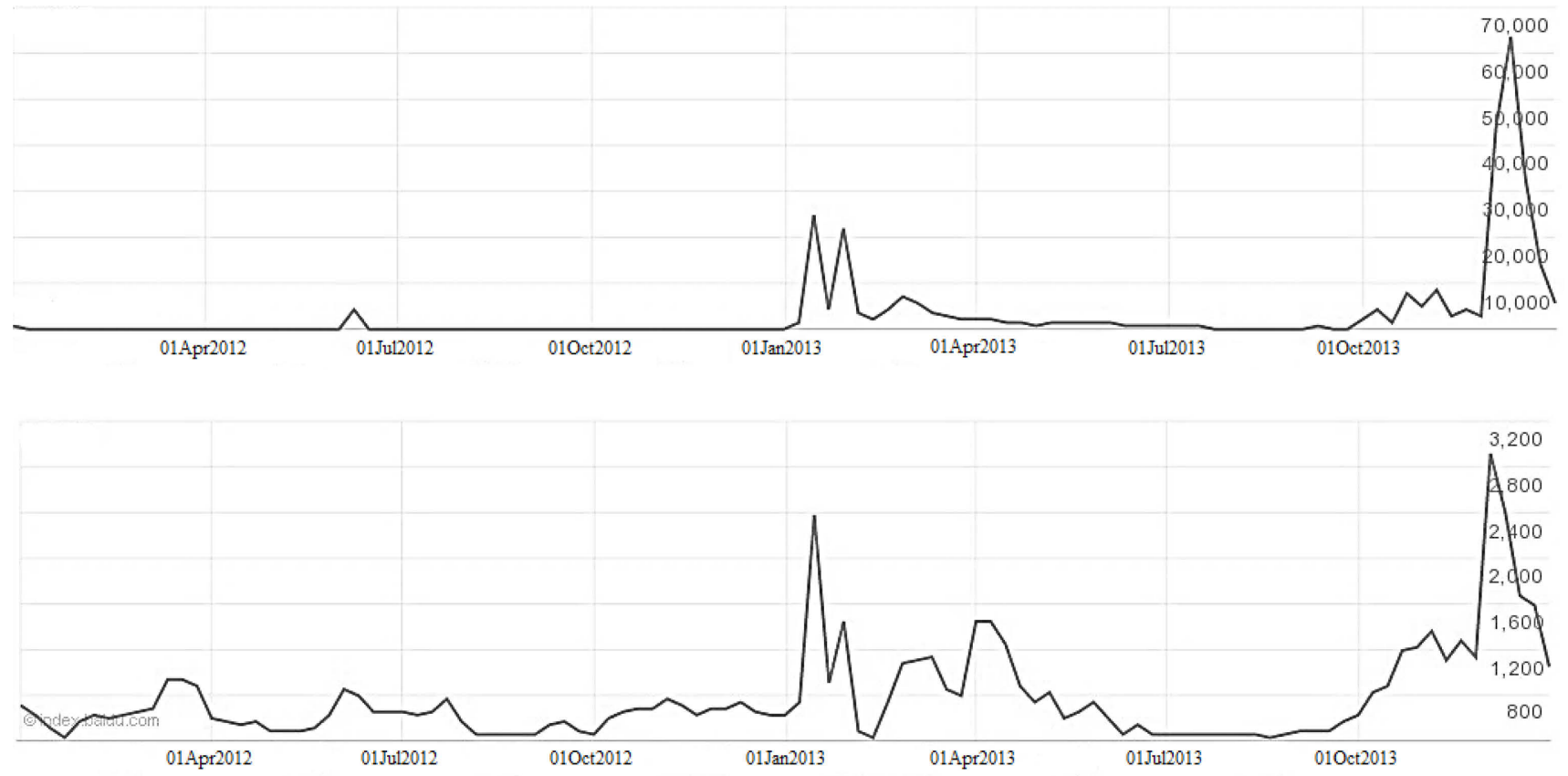

Source: Baidu (2012-2013).

Note: $\quad$ Apr=April; Jan=January; Jul=July; Oct=October 
Figure A.6 Daily API in China, 2010-2012
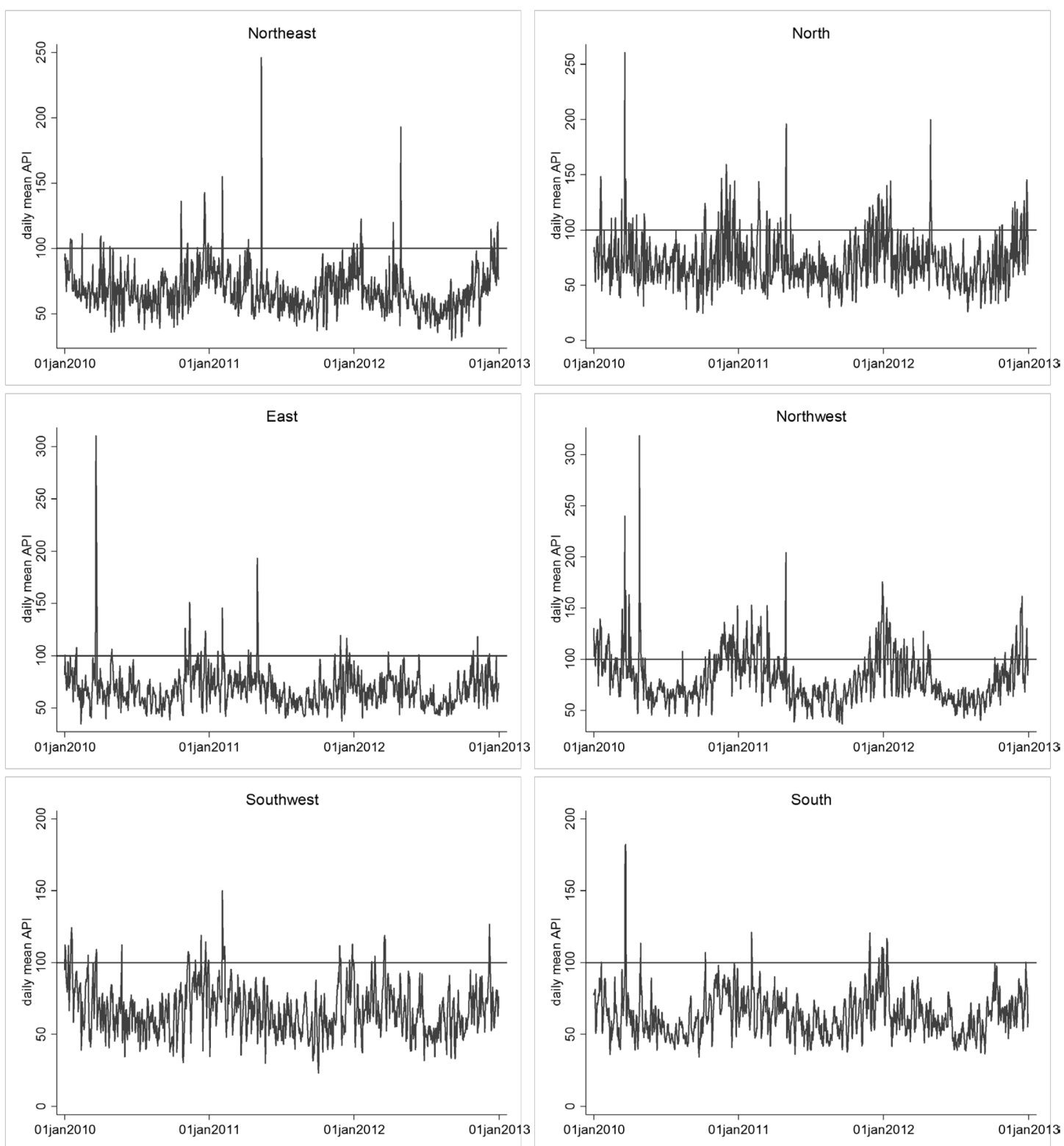

Source: Ministry of Environmental Protection of China (2010-2012).

Note: $\quad$ API = air pollution index; jan = January. The daily mean API is calculated by the weighted average values of all the API report cities within the region, where the weight is the yearly population in each city. The US National Ambient Air Quality Standards of fine particulate matter smaller than 10 micrometers is $0.15 \mathrm{mg} / \mathrm{m}^{3}$, which corresponds to 100 of API in China. Northeast China includes Heilongjiang, Jilin, and Liaoning. North China includes Beijing, Hebei, Inner Mongolia, Shanxi, and Tianjin. East China includes Anhui, Fujian, Jiangsu, Jiangxi, Shandong, Shanghai, and Zhejiang. Northwest China includes Gansu, Ningxia, Qinghai, Shanxi, and Xinjiang. Southwest China includes Guizhou, Sichuan, Tibet, Yunnan, and Chongqing. South China includes Guangdong, Guangxi, Hainan, Henan, Hubei, and Hunan. 
Table A.1 Heterogeneity test of air quality effect, by gender and age

\begin{tabular}{|c|c|c|c|c|c|c|}
\hline \multicolumn{7}{|c|}{ A. Hedonic happiness } \\
\hline Dependent variable & \multicolumn{3}{|c|}{ Male } & \multicolumn{3}{|c|}{ Female } \\
\hline Hedonic happiness & $\begin{array}{l}\text { Young } \\
(16-39)\end{array}$ & $\begin{array}{l}\text { Middle } \\
(40-59)\end{array}$ & $\begin{array}{c}\text { Old } \\
\text { (60 and older) }\end{array}$ & $\begin{array}{l}\text { Young } \\
(16-39)\end{array}$ & $\begin{array}{l}\text { Middle } \\
(40-59)\end{array}$ & $\begin{array}{c}\text { Old } \\
\text { (60 and older) }\end{array}$ \\
\hline & (1) & $(2)$ & (3) & (4) & (5) & (6) \\
\hline Dependent variable mean & 2.937 & 2.904 & 2.900 & 2.900 & 2.763 & 2.751 \\
\hline Visibility & $\begin{array}{c}0.740 \\
(1.209)\end{array}$ & $\begin{array}{c}1.638 \\
(1.229)\end{array}$ & $\begin{array}{c}0.405 \\
(1.200)\end{array}$ & $\begin{array}{l}1.815^{\star *} \\
(0.836)\end{array}$ & $\begin{array}{l}2.007^{\star *} \\
(0.904)\end{array}$ & $\begin{array}{l}3.532^{* *} \\
(1.719)\end{array}$ \\
\hline Sunshine & $\begin{array}{c}0.609 \\
(1.883)\end{array}$ & $\begin{array}{l}-0.386 \\
(2.162)\end{array}$ & $\begin{array}{l}-1.434 \\
(1.996)\end{array}$ & $\begin{array}{l}-0.685 \\
(1.713)\end{array}$ & $\begin{array}{l}2.088 \\
(1.540)\end{array}$ & $\begin{array}{c}0.662 \\
(3.120)\end{array}$ \\
\hline Visibility $\times$ sunshine & $\begin{array}{l}-0.239 \\
(0.488)\end{array}$ & $\begin{array}{l}-0.627 \\
(0.492)\end{array}$ & $\begin{array}{l}-0.154 \\
(0.474)\end{array}$ & $\begin{array}{r}-0.666^{*} \\
(0.348)\end{array}$ & $\begin{array}{c}-0.763^{* *} \\
(0.363)\end{array}$ & $\begin{array}{l}-1.345^{*} \\
(0.687)\end{array}$ \\
\hline Observations & 1,667 & 3,154 & 1,878 & 1,840 & 3,445 & 1,698 \\
\hline$R$-squared & .229 & .165 & .204 & .210 & .171 & .215 \\
\hline \multicolumn{7}{|c|}{ B. Mental well-being } \\
\hline Dependent variable & & Male & & & Female & \\
\hline Percentile of the & $\begin{array}{c}\text { Young } \\
(16-39)\end{array}$ & $\begin{array}{l}\text { Middle } \\
(40-59) \\
\end{array}$ & $\begin{array}{c}\text { Old } \\
\text { (60 and older) }\end{array}$ & $\begin{array}{c}\text { Young } \\
(16-39)\end{array}$ & $\begin{array}{l}\text { Middle } \\
(40-59)\end{array}$ & $\begin{array}{c}\text { Old } \\
\text { (60 and older) }\end{array}$ \\
\hline & (1) & $(2)$ & (3) & (4) & (5) & (6) \\
\hline Dependent variable mean & 0.447 & 0.449 & 0.449 & 0.490 & 0.515 & 0.526 \\
\hline Visibility & $\begin{array}{l}-0.178 \\
(0.389)\end{array}$ & $\begin{array}{c}-0.606^{*} \\
(0.309)\end{array}$ & $\begin{array}{l}-0.717 \\
(0.451)\end{array}$ & $\begin{array}{l}-0.347 \\
(0.555)\end{array}$ & $\begin{array}{c}-0.563^{* *} \\
(0.261)\end{array}$ & $\begin{array}{c}-1.053^{* * *} \\
(0.376)\end{array}$ \\
\hline Sunshine & $\begin{array}{c}0.760 \\
(0.498)\end{array}$ & $\begin{array}{c}0.083 \\
(0.456)\end{array}$ & $\begin{array}{l}-0.803 \\
(0.569)\end{array}$ & $\begin{array}{c}0.635 \\
(0.583)\end{array}$ & $\begin{array}{c}0.215 \\
(0.352)\end{array}$ & $\begin{array}{l}-1.158^{*} \\
(0.610)\end{array}$ \\
\hline Visibility $\times$ sunshine & $\begin{array}{c}0.062 \\
(0.153)\end{array}$ & $\begin{array}{l}0.244^{* *} \\
(0.122)\end{array}$ & $\begin{array}{c}0.281 \\
(0.176)\end{array}$ & $\begin{array}{c}0.130 \\
(0.220)\end{array}$ & $\begin{array}{l}0.221^{\star *} \\
(0.103)\end{array}$ & $\begin{array}{l}0.427^{* * *} \\
(0.147)\end{array}$ \\
\hline $\begin{array}{l}\text { Observations } \\
\text { (Within) } R \text {-squared }\end{array}$ & $\begin{array}{c}3,592 \\
.078\end{array}$ & $\begin{array}{c}6,278 \\
.050\end{array}$ & $\begin{array}{c}3,434 \\
.030\end{array}$ & $\begin{array}{c}3,958 \\
.062\end{array}$ & $\begin{array}{c}6,789 \\
.037\end{array}$ & $\begin{array}{c}3,019 \\
.056\end{array}$ \\
\hline
\end{tabular}

Source: Panel A is from the China Family Panel Studies (ISSS 2012) and panel B is from China Family Panel Studies (ISSS 2010; 2012).

Note: $\quad$ CES-D = Center for Epidemiologic Studies Depression scale. Lower percentile of the CES-D score indicates better mental well-being. In panel A, other covariates and fixed effects are the same as those in column (4) of Table 4.2. In panel B, other covariates and fixed effects are the same as those in column (4) of Table 4.3. Robust standard errors, clustered at the county level, are presented in parentheses. $* 10 \%$ significance level. $* * 5 \%$ significance level. $* * * 1 \%$ significance level. 
Table A.2 Summary statistics of subjective well-being in China, 1990-2012

\begin{tabular}{|c|c|c|c|c|c|c|c|c|}
\hline \multirow[b]{3}{*}{ Year } & \multicolumn{4}{|c|}{ World Values Survey } & \multirow{2}{*}{\multicolumn{2}{|c|}{$\begin{array}{c}\text { CCTV Postcard Survey } \\
\text { Happiness } \\
(1-5) \\
\end{array}$}} & \multirow{2}{*}{\multicolumn{2}{|c|}{$\begin{array}{c}\text { Horizon Research } \\
\text { Urban Life Satisfaction (1-5) }\end{array}$}} \\
\hline & \multicolumn{2}{|c|}{$\begin{array}{l}\text { Life Satisfaction } \\
(1-10)\end{array}$} & \multicolumn{2}{|r|}{$\begin{array}{c}\text { Happiness } \\
(1-4)\end{array}$} & & & & \\
\hline & Mean & Standard deviation & Mean & Standard deviation & Mean & Standard deviation & Mean & Standard deviation \\
\hline 1990 & 7.292 & 2.101 & 2.946 & 0.813 & & & & \\
\hline 1995 & 6.833 & 2.418 & 3.052 & 0.659 & & & & \\
\hline 1997 & & & & & & & 3.69 & \\
\hline 1998 & & & & & & & 3.48 & \\
\hline 1999 & & & & & & & 3.44 & \\
\hline 2000 & & & & & & & 3.27 & 1.05 \\
\hline 2001 & 6.530 & 2.468 & 2.868 & 0.634 & & & 3.28 & 1.13 \\
\hline 2002 & & & & & & & 3.33 & 1.12 \\
\hline 2003 & & & & & & & 3.26 & 1.03 \\
\hline 2004 & & & & & & & 3.38 & 1.01 \\
\hline 2005 & & & & & & & 3.28 & 0.84 \\
\hline 2006 & & & & & & & 3.52 & 0.86 \\
\hline 2007 & 6.760 & 2.395 & 2.936 & 0.749 & 3.623 & 0.921 & 3.35 & 0.84 \\
\hline 2008 & & & & & 3.623 & 0.973 & 3.51 & 0.74 \\
\hline 2009 & & & & & 3.560 & 0.964 & 3.47 & 0.72 \\
\hline 2010 & & & & & 3.477 & 1.008 & 3.41 & 0.79 \\
\hline 2011 & & & & & 3.396 & 0.983 & 3.53 & 0.76 \\
\hline 2012 & 6.858 & 1.985 & 3.006 & 0.585 & 3.477 & 1.001 & 3.57 & 0.69 \\
\hline
\end{tabular}

Source: World Values Survey (1990; 1995; 2001; 2007; 2012), China Central Television (CCTV) Postcard Survey (2007-2013), and Horizon Research Consultancy Group (19972013).

Note: World Values Survey_Life satisfaction: All things considered, how satisfied are you with your life as a whole these days? ([dissatisfied] 12345678910 [satisfied]). Happiness: Taking all things together, would you say you are: very happy, quite happy, not very happy, or not at all happy? (coded 4, 3, 2, or 1). CCTV Postcard Survey (in Chinese)-Happiness: How do you feel about your current life? (very happy, fairly happy, just so-so, not happy, or very unhappy; coded 5, 4, 3, 2, or 1). Horizon (in Chinese) - Life satisfaction: In general, are you satisfied with your current life? (very satisfied, fairly satisfied, average, fairly dissatisfied, or very dissatisfied; coded 5, 4 , 3,2 , or 1 ). 
Table A.3 Placebo test: Air visibility forwarded by a week

\begin{tabular}{|c|c|c|c|c|c|}
\hline Dependent variable & $\begin{array}{c}\text { Life } \\
\text { satisfaction } \\
\text { (1) }\end{array}$ & $\begin{array}{c}\text { Hedonic } \\
\text { happiness } \\
\text { (2) }\end{array}$ & $\begin{array}{c}\text { Mental } \\
\text { well-being } \\
\text { (3) }\end{array}$ & $\begin{array}{l}\text { Depressive } \\
\text { symptoms } \\
\text { (4) }\end{array}$ & $\begin{array}{c}\text { Severe } \\
\text { depression } \\
\text { (5) }\end{array}$ \\
\hline Air quality_F7 & $\begin{array}{c}0.349 \\
(0.820)\end{array}$ & $\begin{array}{l}2.000^{\star *} \\
(1.008)\end{array}$ & $\begin{array}{l}-0.246 \\
(0.291)\end{array}$ & $\begin{array}{l}-0.456 \\
(0.526)\end{array}$ & $\begin{array}{l}-0.049 \\
(0.291)\end{array}$ \\
\hline Sunshine_F7 & $\begin{array}{c}0.503 \\
(1.150)\end{array}$ & $\begin{array}{c}0.829 \\
(1.199)\end{array}$ & $\begin{array}{c}0.277 \\
(0.383)\end{array}$ & $\begin{array}{c}0.083 \\
(0.655)\end{array}$ & $\begin{array}{c}0.170 \\
(0.394)\end{array}$ \\
\hline Air quality_F7×Sunshine_F7 & $\begin{array}{l}-0.116 \\
(0.318)\end{array}$ & $\begin{array}{l}-0.766^{*} \\
(0.400)\end{array}$ & $\begin{array}{c}0.098 \\
(0.114)\end{array}$ & $\begin{array}{c}0.181 \\
(0.207)\end{array}$ & $\begin{array}{c}0.020 \\
(0.116)\end{array}$ \\
\hline Household per capita income & $\begin{array}{l}0.023^{*} \\
(0.012)\end{array}$ & $\begin{array}{c}0.055^{\star * *} \\
(0.010)\end{array}$ & $\begin{array}{c}0.001 \\
(0.004)\end{array}$ & $\begin{array}{c}-0.024^{* * *} \\
(0.005)\end{array}$ & $\begin{array}{c}-0.016^{* * *} \\
(0.004)\end{array}$ \\
\hline Relative income & $\begin{array}{c}0.202^{* * *} \\
(0.012)\end{array}$ & $\begin{array}{c}0.065^{\star * *} \\
(0.009)\end{array}$ & $\begin{array}{c}-0.017^{* * *} \\
(0.003)\end{array}$ & $\begin{array}{c}-0.037^{* * *} \\
(0.005)\end{array}$ & $\begin{array}{c}-0.027^{* * *} \\
(0.004)\end{array}$ \\
\hline Age $(\div 10)$ & & $\begin{array}{c}-0.076^{* *} \\
(0.037)\end{array}$ & & $\begin{array}{c}0.059^{* * *} \\
(0.017)\end{array}$ & $\begin{array}{c}0.054^{* * *} \\
(0.012)\end{array}$ \\
\hline Age $(\div 10)$ squared & $\begin{array}{c}0.027 \\
(0.019)\end{array}$ & $\begin{array}{l}0.006^{*} \\
(0.004)\end{array}$ & $\begin{array}{c}0.034^{* * *} \\
(0.008)\end{array}$ & $\begin{array}{c}-0.005^{* * *} \\
(0.002)\end{array}$ & $\begin{array}{c}-0.004^{* * *} \\
(0.001)\end{array}$ \\
\hline Male & & $\begin{array}{c}0.073^{* * *} \\
(0.015)\end{array}$ & & $\begin{array}{c}-0.078^{* * *} \\
(0.008)\end{array}$ & $\begin{array}{c}-0.054^{* * *} \\
(0.006)\end{array}$ \\
\hline Married & $\begin{array}{l}0.112^{*} \\
(0.066)\end{array}$ & $\begin{array}{c}0.108^{* * *} \\
(0.028)\end{array}$ & $\begin{array}{c}-0.069^{* * *} \\
(0.016)\end{array}$ & $\begin{array}{c}-0.115^{* * *} \\
(0.014)\end{array}$ & $\begin{array}{c}-0.092^{* * *} \\
(0.011)\end{array}$ \\
\hline Divorced & $\begin{array}{l}-0.247^{*} \\
(0.148)\end{array}$ & $\begin{array}{l}-0.105^{\star} \\
(0.059)\end{array}$ & $\begin{array}{c}0.029 \\
(0.039)\end{array}$ & $\begin{array}{c}0.041 \\
(0.034)\end{array}$ & $\begin{array}{l}-0.007 \\
(0.025)\end{array}$ \\
\hline Education & $\begin{array}{c}0.005 \\
(0.006)\end{array}$ & $\begin{array}{c}0.015^{* * *} \\
(0.003)\end{array}$ & $\begin{array}{c}0.000 \\
(0.002)\end{array}$ & $\begin{array}{c}-0.010^{* * *} \\
(0.001)\end{array}$ & $\begin{array}{c}-0.006^{* * *} \\
(0.001)\end{array}$ \\
\hline Unemployed & $\begin{array}{l}-0.036 \\
(0.058)\end{array}$ & $\begin{array}{l}-0.102 \\
(0.080)\end{array}$ & $\begin{array}{l}0.028^{* *} \\
(0.013)\end{array}$ & $\begin{array}{c}0.103^{* * *} \\
(0.033)\end{array}$ & $\begin{array}{c}0.000 \\
(0.023)\end{array}$ \\
\hline State employee & $\begin{array}{c}0.034 \\
(0.048)\end{array}$ & $\begin{array}{l}-0.033 \\
(0.027)\end{array}$ & $\begin{array}{l}-0.002 \\
(0.012)\end{array}$ & $\begin{array}{l}-0.005 \\
(0.012)\end{array}$ & $\begin{array}{c}0.012 \\
(0.010)\end{array}$ \\
\hline Party & $\begin{array}{c}0.077 \\
(0.065)\end{array}$ & $\begin{array}{c}0.112^{* * *} \\
(0.031)\end{array}$ & $\begin{array}{l}-0.014 \\
(0.020)\end{array}$ & $\begin{array}{c}-0.037^{* * *} \\
(0.012)\end{array}$ & $\begin{array}{c}-0.029^{\star * *} \\
(0.009)\end{array}$ \\
\hline Chronic disease & $\begin{array}{c}-0.086^{* * *} \\
(0.026)\end{array}$ & $\begin{array}{c}-0.124^{* * *} \\
(0.027)\end{array}$ & $\begin{array}{c}0.043^{* * *} \\
(0.008)\end{array}$ & $\begin{array}{c}0.113^{* \star *} \\
(0.012)\end{array}$ & $\begin{array}{c}0.100^{* * *} \\
(0.011)\end{array}$ \\
\hline Individual fixed effect & Yes & No & Yes & No & No \\
\hline County fixed effect & No & Yes & No & Yes & Yes \\
\hline Weather controls & Yes & Yes & Yes & Yes & Yes \\
\hline $\begin{array}{l}\text { Year, month, day-of-week } \\
\text { fixed effects }\end{array}$ & Yes & Yes & Yes & Yes & Yes \\
\hline Observations & 27,466 & 13,719 & 27,123 & 13,466 & 13,466 \\
\hline$R$-squared & .055 & .148 & .028 & .157 & .124 \\
\hline
\end{tabular}

Source: China Family Panel Studies (ISSS 2010; 2012).

Note: The weather controls include mean temperature and its square, temperature difference (daily maximum-minimum), total precipitation, maximum sustained wind speed, and a dummy for bad weather, and they are all forward seven days. Robust standard errors, clustered at the county level, are presented in parentheses. $* 10 \%$ significance level. $* * 5 \%$ significance level. ***1\% significance level. 


\section{APPENDIX B: CENTER FOR EPIDEMIOLOGIC STUDIES DEPRESSION SCALE (CES-D) IN CHINA FAMILY PANEL STUDIES (CFPS)}

\section{0-item CES-D in CFPS 2012}

Below is a list of the ways you might have felt or behaved. Please tell me how often you have felt this way during the past week.

1. Rarely or None of the Time (Less than 1 Day)

2. Some or a Little of the Time (1-2 Days)

3. Occasionally or a Moderate Amount of Time (3-4 Days)

4. Most or All of the Time (5-7 Days)

During the past week:

1. I was bothered by things that usually don't bother me.

2. I did not feel like eating; my appetite was poor.

3. I felt that I could not shake off the blues even with help from my family or friends.

4. I felt that I was just as good as other people.

5. I had trouble keeping my mind on what I was doing.

6. I felt depressed.

7. I felt that everything I did was an effort.

8. I felt hopeful about the future.

9. I thought my life had been a failure.

10. Ifelt fearful.

11. My sleep was restless.

12. I was happy.

13. I talked less than usual.

14. I felt lonely.

15. People were unfriendly.

16. I enjoyed life.

17. I had crying spells.

18. Ifelt sad.

19. Ifelt that people dislike me.

20. I could not get "going."

\section{Six-item CES-D in CFPS 2010}

Below is a list of the ways you might have felt or behaved. Please tell me how often you have felt this way during the past month.

1. Never

2. Sometimes

3. Half the Time

4. Often

5. Almost Every Day

During the past month:

1. I felt depressed and nothing can cheer me up.

2. I felt nervous.

3. I felt restless and hard to calm down.

4. I felt hopeless about the future.

5. I felt that everything I did was an effort.

6. My life was meaningless. 


\section{APPENDIX C: SUNSHINE DURATION CALCULATION FORMULA}

The formula we use to calculate sunshine duration (hours) is as follows:

$$
\text { sunshinehours }=24 \times \frac{\arccos (\tan \alpha \tan \beta)}{180^{\circ}}
$$

Where $\alpha$ is the latitude of county $j$, and $\beta$ is the latitude of direct sunshine point at date $t$. Noting that $\beta$ is assumed to be positive if the location of the county and the direct sunshine point are on different hemispheres (left figure), while $\beta$ is negative if they are on the same hemisphere (right figure).

\section{Figure C.1 Sunshine duration calculation}
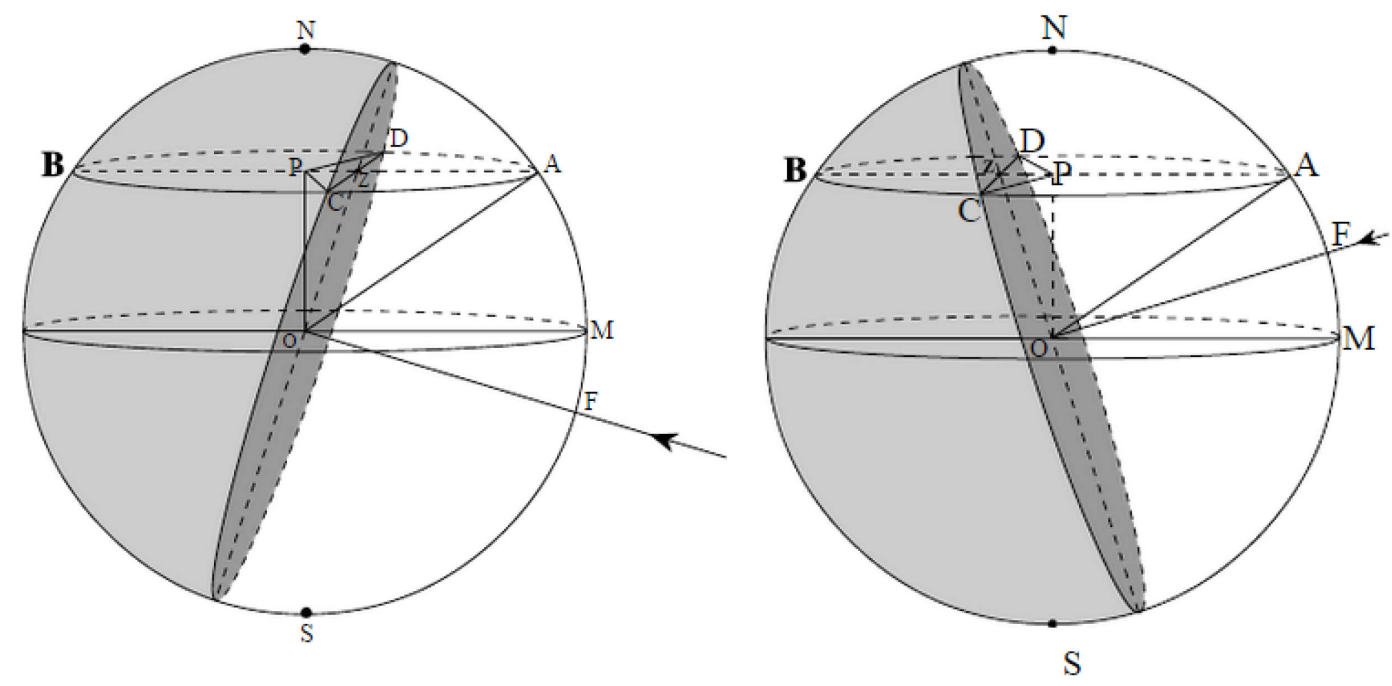

$$
\angle \mathrm{AOM}=\alpha, \angle \mathrm{FOM}=\beta
$$

$\angle \mathrm{AOM}=\alpha, \angle \mathrm{FOM}=-\beta$

Source: Photovoltaic Education Network (2013). 


\section{REFERENCES}

Baidu. Various years. Baidu Index of "Wumai (haze)" and "Kouzhao (mask)". Accessed September 21, 2015. http://index.baidu.com/.

Bailly, D., R. Beuscart, and C. Collinet. 1992. "Sex Differences in the Manifestations of Depression in Young People: A Study of French High School Students." European Child \& Adolescent Psychiatry 1: 135-155.

Beatty, T. K. M., and J. P. Shimshack. 2014. "Air Pollution and Children's Respiratory Health: A Cohort Analysis." Journal of Environmental Economics and Management 67: 39-57.

Chang, T., J. G. Zivin, T. Gross, and M. Neidell. 2014. Particulate Pollution and the Productivity of Pear Packers. NBER Working Paper 19944. Cambridge, MA, US: National Bureau of Economic Research.

Chen, X. 2015a. "Old Age Pension and Intergenerational Living Arrangements: A Regression Discontinuity Design." Review of Economics of the Household, forthcoming.

_. 2015b. "Relative Deprivation and Individual Well-being: Health, Human Capital and Happiness." IZA World of Labor 140: 1-10.

Chen, X., H. Deng, and Y. Zhang. 2015. Air Pollution and Fetal Development: Evidence from Prenatal Ultrasound Scans. Working paper. New Haven, CT, US: Yale University.

Chen, Y., A. Ebenstein, M. Greenstone, and H. Li. 2013. "Evidence on the Impact of Sustained Exposure to Air Pollution on Life Expectancy from China's Huai River Policy." PNAS 110: 12936-12941.

Chen, Y., G. Z. Jin, N. Kumar, and G. Shi. 2012. "Gaming in Air Pollution Data? Lessons from China." B.E. Journal of Economic Analysis \& Policy 13 (3): 1-43.

Cheung, H.-C., T. Wang, K. Baumann, and H. Guo. 2005. "Influence of Regional Pollution Outflow on the Concentrations of Fine Particulate Matter and Visibility in the Coastal Area of Southern China." Atmospheric Environment 39: 6463-6474.

China Central Television (CCTV) Postcard Survey. 2007-2013. http://jingji.cntv.cn/special/jjshdde/

Clark, A. E., P. Frijters, and M. A. Shields. 2008. "Relative Income, Happiness, and Utility: An Explanation for the Easterlin Paradox and Other Puzzles." Journal of Economic Literature 46: 95-144.

Cohen, A. J., H. Ross Anderson, B. Ostro, K. D. Pandey, M. Krzyzanowski, N. Künzli, K. Gutschmidt, A. Pope, I. Romieu, J. M. Samet, and K. Smith. 2005. "The Global Burden of Disease due to Outdoor Air Pollution." Journal of Toxicology and Environmental Health, Part A: Current Issues 68 (13/14): 1301-1307.

Cunningham, M. R. 1979. "Weather, Mood, and Helping Behavior: Quasi Experiments with the Sunshine Samaritan." Journal of Personality and Social Psychology 37 (11): 1947-1956.

Deaton, A., and A. A. Stone. 2013. “Two Happiness Puzzles." American Economic Review 103: 591-597.

Deng, X., X. Tie, D. Wu, X. Zhou, X. Bi, H. Tan, F. Li, and C. Jiang. 2008. "Long-term Trend of Visibility and Its Characterizations in the Pearl River Delta Region, China." Atmospheric Environment 42: 1424-1435.

Di Tella, R., and R. MacCulloch. 2006. "Some Uses of Happiness Data in Economics.” Journal of Economic Perspectives 20 (1): 25-46.

Easterlin, R. A. 1974. "Does Economic Growth Improve the Human Lot? Some Empirical Evidence.” In Nations and Households in Economic Growth, edited by P. A. David and M. W. Reder. New York: Academic Press.

1995. "Will Raising the Incomes of All Increase the Happiness of All?" Journal of Economic Behavior and Organization 27: 35-47.

Easterlin, R. A., L. Angelescu McVey, M. Switek, O. Sawangfa, and J. Smith Zweig. 2010. "The Happiness-income Paradox Revisited." PNAS 107 (52): 22463-22468.

Easterlin, R. A., R. Morgan, M. Switek, and F. Wang. 2012. “China's Life Satisfaction, 1990-2010.” PNAS 109: 9775-9780. 
Ferreira, S., A. Akay, F. Brereton, J. Cuñado, P. Martinsson, M. Moro, and T. F. Ningal. 2013. "Life Satisfaction and Air Quality in Europe." Ecological Economics 88: 1-10.

Frederick, S., and G. Loewenstein. 1999. "Hedonic Adaptation." In Scientific Perspectives on Enjoyment, Suffering, and Well-being, edited by D. Kahneman, E. Diener, and N. Schwarz, 302-329. New York, US: Russell Sage Foundation.

Gallagher, P., W. Lazarus, H. Shapouri, R. Conway, F. Bachewe, and A. Fischer. 2010. "Cardiovascular DiseaseRisk Benefits of Clean Fuel Technology and Policy: A Statistical Analysis.” Energy Policy 38: 1210-1222.

Ghanem, D., and J. Zhang. 2014. “Effortless Perfection': Do Chinese Cities Manipulate Air Pollution Data?” Journal of Environmental Economics and Management 68: 203-225.

Graham, C., S. Chattopadhyay, and M. Picon. 2010. "The Easterlin and Other Paradoxes: Why Both Sides of the Debate May Be Correct?” In International Differences in Well-being, edited by E. Diener, D. Kahneman, and J. Helliwell, 247-290. Oxford, UK: Oxford University Press.

Greenstone, M., and R. Hanna. 2014. "Environmental Regulations, Air and Water Pollution, and Infant Mortality in India." American Economic Review 104 (10): 3038-3072.

Hagerty, M. R., and R. Veenhoven. 2003. "Wealth and Happiness Revisited—Growing National Income Does Go with Greater Happiness." Social Indicators Research 64 (1): 1-27.

Helliwell, J. 2012. Understanding and Improving the Social Context of Well-being. NBER Working Paper 18486. Cambridge, MA, US: National Bureau of Economic Research.

Horizon Research Consultancy Group. 1997-2013. http://www.horizon-china.com/

Inglehart, R., R. Foa, C. Peterson, and C. Welzel. 2008. "Development, Freedom, and Rising Happiness: A Global Perspective (1981-2007)." Perspectives on Psychological Science 3 (4): 264-285.

ISSS (Institute of Social Science Survey). Various years. China Family Panel Studies. Accessed September 2, 2015. www.isss.edu.cn/cfps/EN/.

Kahneman, D., and A. Deaton. 2010. "High Income Improves Evaluation of Life but Not Emotional Well-being." PNAS 107: 16489-16493.

Knight, J., and R. Gunatilaka. 2010. “The Rural-urban Divide in China: Income but Not Happiness?” Journal of Development Studies 46: 506-534. 2011. "Does Economic Growth Raise Happiness in China?" Oxford Development Studies 39: 1-24.

Knight, J., L. Song, and R. Gunatilaka. 2009. "Subjective Well-being and Its Determinants in Rural China." China Economic Review 20: 635-649.

Lavy, V., A. Ebenstein, and S. Roth. 2014a. The Impact of Short Term Exposure to Ambient Air Pollution on Cognitive Performance and Human Capital Formation. NBER Working Paper 20648. Cambridge, MA, US: National Bureau of Economic Research.

2014b. The Long Run Human Capital and Economic Consequences of High-stakes Examinations. NBER Working Paper 20647. Cambridge, MA, US: National Bureau of Economic Research.

Lee, Y. L., and R. Sequeira. 2001. "Visibility Degradation across Hong Kong: Its Components and Their Relative Contributions." Atmospheric Environment 35: 5861-5872.

Levinson, A. 2012. "Valuing Public Goods Using Happiness Data: The Case of Air Quality." Journal of Public Economics 96: 869-880.

Li, J., C. Xia, and X. Yu. 2014. "Jiating Yiliao Zhichu yu Fudan.” In China Minsheng Fazhan Baogao, edited by Y. Xie, X. Zhang, and J. Li. Zurich: Peking University Press.

Li, T., H. Liu, and A. Salvo. 2015. "Severe Air Pollution and Labor Productivity." Accessed June 14, 2015. http://papers.ssrn.com/sol3/papers.cfm?abstract id=2581311.

Luechinger, S. 2009. "Valuing Air Quality Using the Life Satisfaction Approach.” Economic Journal 119: 482-515. 
- 2010. "Life Satisfaction and Transboundary Air Pollution.” Economics Letters 107: 4-6.

Luttmer, E. 2005. "Neighbors as Negative: Relative Earnings and Well-being." Quarterly Journal of Economics 120 (3): 963-1002.

MacKerron, G., and S. Mourato. 2009. “Life Satisfaction and Air Quality in London.” Ecological Economics 68: 1441-1453.

Malm, W. C. 1999. Introduction to Visibility. Fort Collins, CO, US: Cooperative Institute for Research in the Atmosphere (CIRA), NPS Visibility Program, Colorado State University.

Mearns, E. W., and C. H. Best. 2013. "Strong Coherence between Cloud Cover and Surface Temperature Variance in the UK." Accessed June 14, 2015. www.euanmearns.com/wp-content/uploads/2013/11/ 2348370 merged 1377705028.pdf.

Menz, T. 2011. "Do People Habituate to Air Pollution? Evidence from International Life Satisfaction Data." Ecological Economics 71: 211-219.

Ministry of Environmental Protection of China. Various years. “Air Quality Daily”. Accessed September 23, 2015. http://datacenter.mep.gov.cn/report/air daily/air dairy en.jsp.

National Oceanic and Atmospheric Administration. Various years. Visibility Data. ftp://ftp.ncdc.noaa.gov/.

Oswald, A. J. 1997. “Happiness and Economic Performance.” Economic Journal 107: 1815-1831.

Photovoltaic Education Network. 2013. “Calculation of Solar Insolation.” Accessed September 23, 2015. www.pveducation.org/pvcdrom/properties-of-sunlight/calculation-of-solar-insolation/.

Qiu, J., and L. Yang. 2000. "Variation Characteristics of Atmospheric Aerosol Optical Depths and Visibility in North China during 1980-1994." Atmospheric Environment 34: 603-609.

Radloff, L. S. 1977. "The CES-D Scale: A Self-report Depression Scale for Research in the General Population.” Applied Psychological Measurement 1: 385-401.

Rehdanz, K., and D. Maddison. 2008. "Local Environmental Quality and Life-satisfaction in Germany.” Ecological Economics 64: 787-797.

Stevenson, B., and J. Wolfers. 2008. Economic Growth and Subjective Well-being: Reassessing the Easterlin Paradox. NBER Working Paper 14282. Cambridge, MA, US: National Bureau of Economic Research.

Stone, A. A., and C. Mackie, eds. 2014. Subjective Well-being: Measuring Happiness, Suffering, and Other Dimensions of Experience. Washington, DC: National Research Council, National Academies Press.

Tanaka, S. 2015. "Environmental Regulations on Air Pollution in China and Their Impact on Infant Mortality." Journal of Health Economics 42: 90-103.

Weir, K. 2012. “Smog in Our Brains.” Monitor on Psychology 43 (7): 32.

Welsch, H. 2006. "Environment and Happiness: Valuation of Air Pollution Using Life Satisfaction Data." Ecological Economics 58: 801-813. 2007. “Environmental Welfare Analysis: A Life Satisfaction Approach.” Ecological Economics 62: 544551.

Wolfson, E. 2013. "Your Zodiac Sign, Your Health.” The Atlantic, November, 15.

World Values Survey. Various years. "Data and Documentation”. Accessed September 23, 2015. www.worldvaluessurvey.org/wvs.jsp.

Xie, Y., X. Zhang, Q. Xu, and C. Zhang. 2013. “Shouru Fenpei.” In China Minsheng Fazhan Baogao, edited by Y. Xie, X. Zhang, and J. Li. Zurich, Switzerland: Peking University Press.

Zhang, C., Q. Xu, X. Zhou, X. Zhang, and Y. Xie. 2014. “Are Poverty Rates Underestimated in China? New Evidence from Four Recent Surveys.” China Economic Review 31: 410-425.

Zhang, X., J. Yang, and S. Wang. 2011. "China Has Reached the Lewis Turning Point.” China Economic Review 22 (4): 542-554. 





\section{RECENT IFPRI DISCUSSION PAPERS}

\section{For earlier discussion papers, please go to www.ifpri.org/pubs/pubs.htm\#dp. All discussion papers can be downloaded free of charge.}

1462. The impact of the use of new technologies on farmers' wheat yield in Ethiopia: Evidence from a randomized controlled trial. Gashaw Tadesse Abate, Alan de Brauw, Nicholas Minot, and Tanguy Bernard, 2015.

1461. Living like there's no tomorrow: Saving and spending following the Sichuan Earthquake. Mateusz Filipski, Ling Jin, Xiaobo Zhang, and Kevin Chen, 2015.

1460. Agricultural value chain development in practice: Private sector-led smallholder Development. Shashidhara Kolavalli, Akwasi Mensah-Bonsu, and Saima Zaman, 2015.

1459. Cropping Intensity gaps: The potential for expanding global harvest areas. Wenbin Wu, Liangzhi You, and Kevin Chen, 2015.

1458. Migration, gender, and farming systems in Asia: Evidence, data, and knowledge gaps. Valerie Mueller, Chiara Kovarik, Kathryn Sproule, and Agnes Quisumbing, 2015.

1457. Los efectos de la roya en las economías Centroamericanas. Valeria Piñeiro, Samuel Morley, and Pablo Elverdin, 2015.

1456. Agriculture, gendered time use, and nutritional outcomes: A systematic review. Deborah Johnston, Sara Stevano, Hazel Malapit, Elizabeth Hull, and Suneetha Kadiyala, 2015.

1455. Public benefits of private technology adoption: the localized spatial externalities of water conservation in eastern Uttar Pradesh. Anil K. Bhargava, Travis J. Lybbert, and David J. Spielman, 2015.

1454. Supply-side dynamics of chickpeas and pigeon peas in India. Kalimuthu Inbasekar, Devesh Roy, and P. K. Joshi, 2015.

1453. Measuring women's decisionmaking: Indicator choice and survey design experiments from cash and food transfer evaluations in Ecuador, Uganda, and Yemen. Amber Peterman, Benjamin Schwab, Shalini Roy, Melissa Hidrobo, and Daniel Gilligan, 2015.

1452. The potential of farm-level technologies and practices to contribute to reducing consumer exposure to aflatoxins: $A$ theory of change analysis. Nancy Johnson, Christine Atherstone, and Delia Grace, 2015.

1451. How will training traders contribute to improved food safety in informal markets for meat and milk? A theory of change analysis. Nancy Johnson, John Mayne, Delia Grace, and Amanda Wyatt, 2015.

1450. Communication and coordination: Experimental evidence from farmer groups in Senegal. Fo Kodjo Dzinyefa Aflagah, Tanguy Bernard, and Angelino Viceisza, 2015.

1449. The impact of household health shocks on female time allocation and agricultural labor participation in rural Pakistan. Gissele Gajate-Garrido, 2015.

1448. The biophysical potential for urea deep placement technology in lowland rice production systems of Ghana and Senegal. Cindy Cox, Ho-Young Kwon, and Jawoo Koo, 2015.

1447. The Impact of investment in agricultural research and development and agricultural productivity. Nicostrato D. Perez and Mark W. Rosegrant, 2015.

1446. Agricultural diversification and poverty in India. Pratap S. Birthal, Devesh Roy, and Digvijay S. Negi, 2015.

1445. Peer effects in the valuation of attributes and practices for food safety: Findings from the study of dairy consumers in India. Raj Chandra, Abdul Munasib, Devesh Roy, and Vinay Kumar Sonkar, 2015.

1444. The Bali Agreement: An assessment from the perspective of developing countries. Eugenio Díaz-Bonilla and David Laborde, 2015.

1443. Rural and agricultural mechanization: A history of the spread of small engines in selected Asian countries. Stephen Biggs and Scott Justice, 2015.

1442. Investigating public financial accounts and coding system in Malawi and measuring agricultural expenditures within the system. Chance Mwabutwa, 2015.

1441. The effects of political competition on rural land: Evidence from Pakistan. Katrina Kosec, Hamza Haider, David J. Spielman, and Fatima Zaidi, 2015. 
INTERNATIONAL FOOD POLICY

\section{RESEARCH INSTITUTE}

www.ifpri.org

IFPRI HEADQUARTERS

$2033 \mathrm{~K}$ Street, NW

Washington, DC 20006-1002 USA

Tel.: +1-202-862-5600

Fax: +1-202-467-4439

Email: ifpri@cgiar.org 